\title{
Overlooked Tertiary Sulci Serve as a Meso-Scale Link between Microstructural and Functional Properties of Human Lateral Prefrontal Cortex
}

\author{
${ }^{\circledR}$ Jacob A. Miller, ${ }^{1}{ }^{\circledR}$ Willa I. Voorhies, ${ }^{1,2}{ }^{\circledR}$ Daniel J. Lurie, ${ }^{2}{ }^{\circledR}$ Mark D’Esposito, ${ }^{1,2}$ and ${ }^{\circledR}$ Kevin S. Weiner ${ }^{1,2}$ \\ ${ }^{1}$ Helen Wills Neuroscience Institute, University of California, Berkeley, Berkeley, California 94720, and ${ }^{2}$ Department of Psychology, University of \\ California, Berkeley, Berkeley, California 94720
}

Understanding the relationship between neuroanatomy and function in portions of cortex that perform functions largely specific to humans such as lateral prefrontal cortex (LPFC) is of major interest in systems and cognitive neuroscience. When considering neuroanatomical-functional relationships in LPFC, shallow indentations in cortex known as tertiary sulci have been largely unexplored. Here, by implementing a multimodal approach and manually defining 936 neuroanatomical structures in 72 hemispheres (in both males and females), we show that a subset of these overlooked tertiary sulci serve as a meso-scale link between microstructural (myelin content) and functional (network connectivity) properties of human LPFC in individual participants. For example, the posterior middle frontal sulcus (pmfs) is a tertiary sulcus with three components that differ in their myelin content, resting-state connectivity profiles, and engagement across meta-analyses of 83 cognitive tasks. Further, generating microstructural profiles of myelin content across cortical depths for each pmfs component and the surrounding middle frontal gyrus (MFG) shows that both gyral and sulcal components of the MFG have greater myelin content in deeper compared with superficial layers and that the myelin content in superficial layers of the gyral components is greater than sulcal components. These findings support a classic, yet largely unconsidered theory that tertiary sulci may serve as landmarks in association cortices, as well as a modern cognitive neuroscience theory proposing a functional hierarchy in LPFC. As there is a growing need for computational tools that automatically define tertiary sulci throughout cortex, we share pmfs probabilistic sulcal maps with the field.

Key words: brain mapping; connectivity; functional neuroanatomy; microstructure; morphology; prefrontal cortex

Significance Statement

Lateral prefrontal cortex (LPFC) is critical for functions that are thought to be specific to humans compared with other mammals. However, relationships between fine-scale neuroanatomical structures largely specific to hominoid cortex and functional properties of LPFC remain elusive. Here, we show that these structures, which have been largely unexplored throughout history, surprisingly serve as markers for anatomical and functional organization in human LPFC. These findings have theoretical, methodological, developmental, and evolutionary implications for improved understanding of neuroanatomicalfunctional relationships not only in LPFC, but also in association cortices more broadly. Finally, these findings ignite new questions regarding how morphological features of these neglected neuroanatomical structures contribute to functions of association cortices that are critical for human-specific aspects of cognition.

\footnotetext{
Received Sep. 8, 2020; revised Dec. 22, 2020; accepted Jan. 5, 2021.

Author contributions: J.A.M., M.D., and K.S.W. designed research; J.A.M., W.I.V., and K.S.W. performed research; J.A.M., W.I.V., and D.J.L. analyzed data; J.A.M. and K.S.W. wrote the paper.

This work was supported by (1) startup funds provided by the University of California, Berkeley, and the Helen Wills Neuroscience Institute (to K.S.W.); and (2) National Institutes of Health Grant R01-MH-63901 (to J.A.M., M.D.). We thank Jewelia Yao for help with the manual definition of LPFC sulci.

The authors declare no competing financial interests.

Correspondence should be addressed to Jacob A. Miller at jacob_miller@berkeley.edu.

https://doi.org/10.1523/JNEUROSCI.2362-20.2021

Copyright $\odot 2021$ the authors
}

\section{Introduction}

Understanding how anatomical structures of the brain support functional gradients and networks that perform computations for human-specific aspects of cognition is a major goal in systems and cognitive neuroscience. Of the many anatomical structures to target, lateral prefrontal cortex (LPFC) is expanded in the human brain relative to nonhuman primate species commonly used in neuroscience research, such as rhesus macaques (Semendeferi et al., 2002; Donahue et al., 2018; Barrett et al., 2020), and is particularly important given its central role in cognitive control and goal-directed behavior (Miller and Cohen, 
2001; Szczepanski and Knight, 2014). Major progress has been made in understanding the relationship between the functional organization and the large-scale cortical anatomy of human LPFC. For example, previous findings support a hierarchical functional gradient organized along the rostral-caudal anatomical dimension of LPFC spanning several centimeters (Badre and D'Esposito, 2009; Nee and D’Esposito, 2016; Demirtaş et al., 2019). Beyond this large-scale organization of human LPFC, it is largely unknown whether more fine-grained structuralfunctional relationships exist. Thus, to begin to fill this gap in knowledge, we sought to answer the following question in the present study: do individual differences in fine-grained morphological features of LPFC shed light on microstructural and functional properties of LPFC?

An important morphological feature of cortex is the patterning of the indentations, or sulci. Indeed, $60-70 \%$ of the cortex is buried in sulci, and some sulci serve as landmarks that identify different cortical areas, especially in primary sensory cortices (Van Essen and Dierker, 2007; Zilles et al., 2013). In these cases, merely identifying a sulcus provides functional insight (Hinds et al., 2008). Despite this widely replicated relationship between sulcal morphology and functional representations in primary sensory cortices, much less is known regarding the predictability between shallow tertiary sulci and functional representations in association cortex, especially LPFC. A classic theory proposed by Sanides (1964) hypothesized that the late emergence and protracted development of tertiary sulci may co-occur with microstructural and functional features of association cortices, along with cognitive functions such as sustained attention and "active thinking" (Sanides, 1964) that also develop fully after adolescence (Fisher, 2019).

However, at least two factors have prevented the examination of tertiary sulci relative to anatomical and functional organization in human LPFC. First, tertiary sulci are presently excluded from nearly all published neuroanatomical atlases because classic anatomists could not discriminate tertiary sulci from indentations produced by veins and arteries on the outer surface of the cerebrum in postmortem tissue, which is considered the gold standard of anatomical research (Weiner et al., 2018). Consequently, tertiary sulci within the posterior middle frontal gyrus (MFG) were either undefined in classic atlases or conflated with more anterior structures (Fig. 1; Miller et al., 2020a). Second, the majority of human functional magnetic resonance imaging (fMRI) studies of LPFC implement group analyses on average brain templates. As shown in Figure 1, averaging cortical surfaces together causes tertiary sulci in LPFC to disappear, especially within the posterior MFG.

Here, we implemented a multimodal approach demonstrating that identifying individual sulci in LPFC reveals that the posterior middle frontal sulcus (pmfs) serves as a meso-scale link between microstructural (myelin content) and functional (network connectivity) properties of human LPFC in individual participants. Specifically, after manually labeling LPFC tertiary sulci in 72 hemispheres based on a recently proposed labeling scheme (Petrides and Pandya, 2012; Petrides, 2019), we found that three components of the pmfs are dissociable based on myelin content, resting-state functional connectivity profiles, and cognitive task activations. Moreover, the pmfs shows a distinct microstructural profile of myelin content across cortical depths from the surrounding MFG and distinct functional activations from the intermediate frontal sulcus (imfs). Together, these results not only provide important evidence that individual differences in LPFC sulcal patterning reflect meaningful differences in microstructural and functional properties, but also suggest that the pmfs serves as a bridge to the classic hypothesis by Sanides (1964).

\section{Materials and Methods}

In the sections below, we describe the data used and the analysis methods implemented in three separate sections: (1) the general approach 
and a description of the multimodal datasets that were used; (2) a detailed description of the methodology used for sulcal labeling within individual participants; and (3) the calculation of anatomical and functional metrics.

\section{General approach}

We sought to characterize sulcal morphology at the individual level in the LPFC of the human brain. To implement this process, we manually defined sulci following the most recent and comprehensive proposed labeling of sulci in the frontal lobe (Petrides and Pandya, 2012; Petrides, 2019). As in our prior work (Weiner et al., 2014, 2018), all sulci were defined in native space cortical surfaces and individual hemispheres, which enables the most accurate definition of tertiary sulci within in vivo MRI data.

\section{Multimodal HCP dataset}

We analyzed a subset of the multimodal MRI data available for individual participants from the Human Connectome Project (HCP). We began with the first 5 numerically listed HCP participants and then randomly selected 31 additional human participants from the HCP for a total of 36 individuals (17 female, 19 male; age range, 22-36 years).

Anatomical $\mathrm{T}_{1}$-weighted $\left(\mathrm{T}_{1} \mathrm{w}\right)$ MRI scans $(0.7 \mathrm{~mm}$ voxel resolution) were obtained in native space from the HCP database, along with outputs from the FreeSurfer pipeline slightly modified by the HCP (Dale et al., 1999; Fischl et al., 1999a; Glasser et al., 2013). Maps of the ratio of $\mathrm{T}_{1} \mathrm{~W}$ and $\mathrm{T}_{2}$-weighted $\left(\mathrm{T}_{2} \mathrm{w}\right)$ scans, which is a measure of tissue contrast enhancement related to myelin content, were downloaded as part of the HCP "Structural Extended" release. All additional anatomical metrics, which are detailed in the next section, were calculated on the full-resolution, native FreeSurfer (https://surfer.nmr.mgh.harvard.edu/) meshes (Dale et al., 1999; Fischl et al., 1999a,b).

\section{Anatomical labeling and metrics}

Manual sulcal labeling. Guided by a recent comprehensive proposal for labeling sulci in LPFC (Petrides, 2019), each sulcus was manually defined within each individual hemisphere on the FreeSurfer inflated mesh with tksurfer. The curvature metric in FreeSurfer distinguished the boundaries between sulcal and gyral components, and manual lines were drawn to separate sulcal components based on the proposal by Petrides and colleagues (Amiez and Petrides, 2007; Petrides and Pandya, 2012; Petrides, 2019; Germann and Petrides, 2020), as well as the appearance of sulci across the inflated, pial, and smoothwm surfaces. We maintained the number of components for all tertiary sulci (e.g., the three components of the pmfs) based on the proposal by Petrides and colleagues (Amiez and Petrides, 2007; Petrides and Pandya, 2012; Petrides, 2019; Germann and Petrides, 2020) to test whether each of these sulcal components could be defined in a relatively large sample size $(N=72)$ of in vivo hemispheres. The labels were generated using a two-tiered procedure. The labels were first defined manually by J.A.M. and W.I.V. and then finalized by a neuroanatomist (K.S.W.). All anatomical labels for a given hemisphere were fully defined before any morphological or functional analysis of the sulcal labels was performed. The superior, inferior, posterior, and anterior boundaries of our cortical expanse of interest were the following sulci, respectively: (1) the anterior and posterior components of the superior frontal sulcus; (2) the inferior frontal sulcus; (3) the central sulcus (cs); and (4) the horizontal imfs (imfs-h) and vertical imfs (imfs-v). In each hemisphere, we first labeled the large primary sulci such as the central sulcus before labeling the secondary sulci [e.g., superior frontal sulcus (sfs), inferior frontal sulcus (ifs), imfs], and then we identified the tertiary sulcal components of the pmfs. Primary, secondary, and tertiary labels refer to the time in which the sulci emerge in gestation (Sanides, 1964; Chi et al., 1977; Welker, 1990; Armstrong et al., 1995). An example hemisphere with every sulcus labeled within these boundaries is shown in Figure $2 a$, and the pmfs sulcal components are plotted on each hemisphere in Extended Data Figure 2-1.

Quantification of sulcal depth and surface area. Sulcal depth was calculated from the native meshes generated by the FreeSurfer HCP pipeline. Raw values for sulcal depth (in millimeters) were calculated from the sulcal fundus to the smoothed outer pial surface using a custommodified version of a recently developed algorithm for robust morphological statistics building on the FreeSurfer pipeline (Madan, 2019).
Surface area (in square millimeters) was generated for each sulcus through the mris_anatomical_stats function in FreeSurfer (Dale et al., 1999; Fischl et al., 1999a). We focused on sulcal depth as it is the main measurement that is used to discriminate tertiary sulci from primary and secondary sulci. Specifically, primary sulci are deepest, while tertiary sulci are shallowest, and secondary sulci are in between (Sanides, 1964; Chi et al., 1977; Welker, 1990; Armstrong et al., 1995). We also included surface area as tertiary sulci typically also have a reduced surface area compared with primary and secondary sulci.

Calculating $T_{1} w / T_{2} w$ myelin index along an anterior-posterior dimension in LPFC. To test whether there is a relationship between any of our sulci of interest and myelin content, we used an in vivo proxy of myelination: the $\mathrm{T}_{1} \mathrm{w} / \mathrm{T}_{2} \mathrm{~W}$ maps for each individual hemisphere (Glasser and Van Essen, 2011; Shams et al., 2019). To generate the $T_{1} w / T_{2} w$ maps, two $T_{1}$ - and $T_{2}$-weighted images from each participant were registered together and averaged as part of the HCP processing pipeline (Glasser et al., 2013). The averaging helps to reduce motion-related effects or blurring. Additionally, and as described in the study by Glasser et al. (2013), the $\mathrm{T}_{1} \mathrm{w} / \mathrm{T}_{2} \mathrm{w}$ images were bias corrected for distortion effects with field maps. We averaged the $\mathrm{T}_{1} \mathrm{w} / \mathrm{T}_{2} \mathrm{~W}$ ratio value across each vertex for each sulcus to test whether the pmfs sulcal components are separable based on myelin content (Fig. 3). We further sought to characterize the relationship between morphology and myelin by determining whether there was an anterior-posterior gradient of myelination across individual hemispheres. To do so, we first calculated the minimum geodesic distance of each vertex from the central sulcus. Geodesic distance was calculated on the fiducial surface using algorithms in the pycortex package (Gao et al., 2015). Then, we averaged across the vertices within each sulcus and tested for a linear relationship between average distance from the central sulcus and myelin content. To take advantage of each participant's individual data, we built a mixed linear model (random intercepts) in the lme4 $\mathrm{R}$ package, using sulci and hemisphere as explanatory variables to correlate with average myelin content (Fig. 3).

Sampling $T_{1} w / T_{2} w$ myelin index across cortical depths. To investigate the microstructural profile of the pmfs across cortical layers, we generated nine surfaces from the outermost (pial) to the innermost (white matter) layers in all of the manually labeled hemispheres using an equivolumetric approach (Waehnert et al., 2014). We implemented the equivolume surface algorithm spanning nine cortical depths with the surfacetools Python package that builds on top of FreeSurfer (Dale et al., 1999) outputs (https://github.com/kwagstyl/surface_tools). The high-resolution $\mathrm{T}_{1} \mathrm{w} / \mathrm{T}_{2} \mathrm{w}$ volumetric data in each HCP participant's native anatomical space were then sampled onto each equivolume surface using the FreeSurfer mri_vol2surf function to obtain a value of $\mathrm{T}_{1} \mathrm{w} / \mathrm{T}_{2} \mathrm{w}$ at each cortical depth. The stability of depth profiles of $\mathrm{T}_{1} \mathrm{w} / \mathrm{T}_{2} \mathrm{~W}$ values extracted from individual regions was shown to be highest in the same HCP dataset when using a solution of 14 equivolume surfaces, with stability plateauing when using nine or more equivolume surfaces (Paquola et al., 2019). We compared the mean $\mathrm{T}_{1} \mathrm{w} / \mathrm{T}_{2} \mathrm{~W}$ value across depths for each participant in the manually defined pmfs components and the surrounding middle frontal gyrus [as defined by FreeSurfer parcellations (Destrieux et al., 2010); but with the pmfs components removed]. We then conducted a repeated-measures ANOVA followed by post hoc $t$ tests at each depth to test for differences in myelin content between the pmfs components and the MFG (see Fig. 5). Tests across each of the nine cortical depths were corrected for multiple comparisons at a familywise error (FWE) threshold of $p=0.05 / 9$.

Cross-validation of sulcal location. To quantify the ability to predict the location of each sulcus across participants, we registered all sulcal labels to a common template surface (fsaverage) using cortex-based alignment (Fischl et al., 1999b). Similarity between each transformed individual label and the labels defined on fsaverage was calculated via the Dice coefficient, where $X$ and $Y$ are each label, as follows:

$$
\operatorname{Dice}(X, Y)=2 \mid \frac{X \cap Y \mid}{|X|+|Y|}
$$

The cortex-based alignment algorithm aligns the surfaces based on sulcal depth and curvature metrics. We use the central sulcus as a proxy 
noise ceiling measurement for Dice coefficient values from other frontal sulci because it is a large and deep sulcus and is used in the surface registration algorithm that aligns cortical surfaces across participants (Fischl et al., 1999b).

Sulcal probability maps were calculated to describe the vertices with the highest alignment across participants for a given sulcus. A map was generated for each sulcus by calculating, at each vertex in the fsaverage hemisphere, the number of participants with that vertex labeled as the given sulcus, divided by the total number of participants. To avoid overlap among sulci, we then constrained the probability maps into maximum probability maps (MPMs) by only including vertices where (1) $>33 \%$ of participants included the given sulcal label and (2) the sulcus with the highest value of participant overlap was assigned to a given vertex. In a leave-one-participant out cross-validation procedure, we generated probability maps from $n=35$ participants and registered the probability map to the held-out participant's native cortical surface. This provided a measure of sulcal variability and prediction accuracy (see Fig. 8). This procedure also allows the identification of the pmfs sulcal components within held-out individual participants, reducing the extent of manual labeling necessary to identify this structure in future studies. Finally, the MPMs were used when analyzing metaanalytical functional data (described in the Cognitive component modeling section) and whole-brain population receptive field data (see Fig. 7). The MPMs and code for alignment to new participants will be available on Open Science Framework (OSF) with the publication of this article.

\section{Functional metrics}

Resting-state network connectivity fingerprints. To test whether the three pmfs sulcal components were functionally distinct from one another, we calculated and compared functional connectivity network fingerprints for each sulcus. Resting-state network parcellations for each individual participant were used from the article by Kong et al. (2018), who generated individual network definitions by applying a hierarchical Bayesian network algorithm to produce maps for each of 17 networks (Yeo et al., 2011) in individual HCP participants. These data were calculated in the template HCP fs_LR 32k space. We resampled the network profiles for each participant onto the fsaverage cortical surface and, then, to each native surface using CBIG tools (https://github.com/ ThomasYeoLab/CBIG). We then calculated the overlap of each pmfs sulcus in each participant with each of the 17 resting-state networks. We also separated the components of the pmfs and tested whether they showed similar or different network connectivity fingerprints using a three-way repeated-measures ANOVA (sulcal component $\times$ network $\times$ hemisphere). Variability across individuals in the network profiles for each pmfs component was calculated by generating the Wasserstein metric (Earth Mover's Distance) between the resting-state network overlap values for each unique pair of participants (see Fig. $5 b$ ).

Cognitive component modeling. To further examine whether the posterior pmfs (pmfs-p), intermediate pmfs (pmfs-i), and anterior pmfs (pmfs-a) are functionally distinct, we quantified the overlap between the MPMs of each sulcal component and meta-analytic fMRI data from hundreds of experiments aligned to the fsaverage surface. Specifically, we quantitatively related the sulcal MPMs to vertex-wise maps for 14 cognitive components, which quantify how each vertex is recruited in a given set of cognitive operations across tasks and experiments (Yeo et al., 2015). We used a Bayesian method of expectation maximization to determine the combination of cognitive components that best fit each sulcal MPM. This resulted in a set of probabilities for each cognitive component for each sulcal map. We tested whether all sulci and the three components of the pmfs were distinguishable based on these cognitive component loadings from a repeated-measures ANOVA (see Fig. 6).

Retinotopic response mapping. To determine whether there was any correspondence between the manually labeled LPFC sulci and retinotopic representations, we analyzed a recent population receptive field mapping dataset (Benson et al., 2018). As these data were only available in a template (fsaverage) space, we used the predicted sulcal locations from probabilistic maps (as used in the cognitive components analysis) for these analyses (see Fig. 7). For each sulcus, we extracted the mean $R^{2}$ value (the percentage of variance in each vertex explained by the population receptive field model) across participants for vertices that showed meaningful retinotopic responses (thresholded at $R^{2}>10 \%$; Mackey et al., 2017)

\section{Statistical methods}

All repeated-measures ANOVAs (including sphericity correction) and post hoc $t$ tests were performed with the afex and emmeans $\mathrm{R}$ packages, imported into Python via rpy2. For each repeated-measures ANOVA, cortical hemisphere and sulcus were used as within-subject factors. Effect sizes for each main effect and interaction were calculated and reported with the generalized $\eta$-squared metric (Fritz et al., 2012). Mixed linear models were implemented in the lme4 R package. Cortical surface files were loaded in and operated on in Python using the Nilearn software (https://nilearn.github.io).

\section{Data availability}

Data were provided by the Human Connectome Project, WU-Minn Consortium [Principal Investigators: David Van Essen and Kamil Ugurbil; National Institutes of Health (NIH) Grant 1U54-MH-091657] funded by the $16 \mathrm{NIH}$ Institutes and Centers that support the NIH Blueprint for Neuroscience Research; and by the McDonnell Center for Systems Neuroscience at Washington University]. The HCP dataset and processing have been described in previous publications (Glasser et al., 2013, 2016). The probability maps for LPFC sulcal definitions and analysis code will be freely available with the publication of the paper on OSF (https://osf.io/7fwqk/).

\section{Results}

Before conducting our multimodal examination relating morphological features of tertiary sulci to microstructural and functional properties of LPFC, we first had to confront the contradictory nature of historical and modern definitions of sulci within the MFG. For example, sulcal definitions within the MFG vary in (1) their nomenclature, (2) the number of sulcal components depicted or acknowledged in schematics, (3) the omission or inclusion of sulci within the posterior MFG, and (4) the actual empirical data that is included to support the illustration of the sulcal patterning (Fig. 1). To ameliorate these concerns and to either empirically support or to refute the generality of sulcal definitions within the posterior MFG, we apply a classic, multimodal approach that has been used to distinguish cortical areas from one another to determine sulcal definitions in the posterior MFG. Specifically, after identifying each sulcus within the posterior MFG based on recent proposals (Petrides and Pandya, 2012; Petrides, 2019), we use both anatomical and fMRI data to either support or refute the identification of individual sulci within this cortical expanse. Implementing this two-pronged approach, we first examined whether the three components of the pmfs are consistently identifiable within individual hemispheres. And, if so, we then tested whether the three pmfs components are anatomically and functionally homogenous or serve to identify anatomical and functional heterogeneity in LPFC. This approach supports the latter, in which there are three anatomically and functionally distinct sulci within the posterior MFG: the pmfs-p, the pmfs-i, and the pmfs-a.

\section{Three posterior middle frontal sulci are identifiable within individuals and are characteristically shallow}

Before examining the sulcal patterning within the posterior MFG, we first identified reliable sulci (see the Manual sulcal labeling section in Materials and Methods) surrounding the MFG in both in vivo cortical surface reconstructions of MRI data and postmortem brains (Fig. 2a). Posteriorly, we identified the cs, as well as the superior precentral sulcus (sprs) and inferior 
a sulcal labels

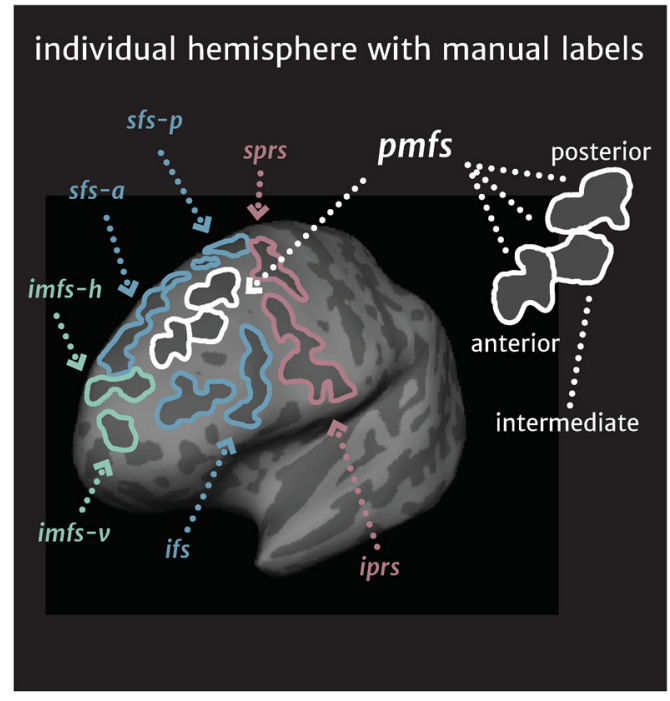

post-mortem hemispheres with manual labels

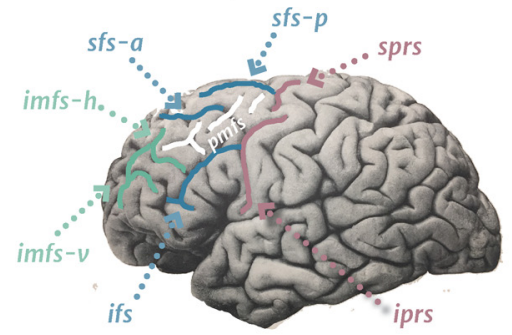

post-mortem sections with manual labels

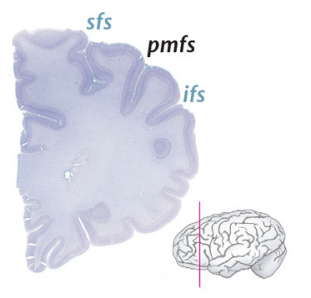

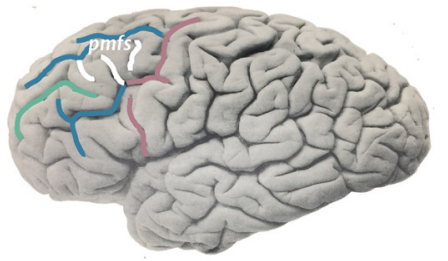

anterior $<\ldots . . . .$.$) posterior$

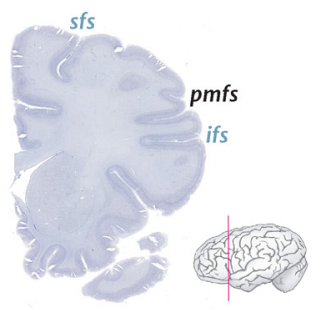

b sulcal morphology

\begin{tabular}{|c|c|}
\hline \multicolumn{2}{|c|}{ lateral frontal sulci } \\
\hline central & CS \\
\hline $\begin{array}{l}\text { inferior } \\
\text { precentral }\end{array}$ & iprs \\
\hline superior precentral & sprs \\
\hline inferior frontal & ifs \\
\hline $\begin{array}{l}\text { posterior middle } \\
\text { frontal, posterior }\end{array}$ & $p m f s-p$ \\
\hline $\begin{array}{l}\text { posterior middle } \\
\text { frontal, intermediate }\end{array}$ & pmfs-i \\
\hline $\begin{array}{l}\text { posterior middle } \\
\text { frontal, anterior }\end{array}$ & $p m f s-a$ \\
\hline $\begin{array}{l}\text { superior frontal, } \\
\text { posterior }\end{array}$ & $s f s-p$ \\
\hline $\begin{array}{l}\text { superior frontal, } \\
\text { anterior }\end{array}$ & $s f s-a$ \\
\hline $\begin{array}{l}\text { intermediate } \\
\text { frontal, horizontal }\end{array}$ & imfs-h \\
\hline $\begin{array}{l}\text { intermediate } \\
\text { frontal, vertical }\end{array}$ & imfs-v \\
\hline
\end{tabular}
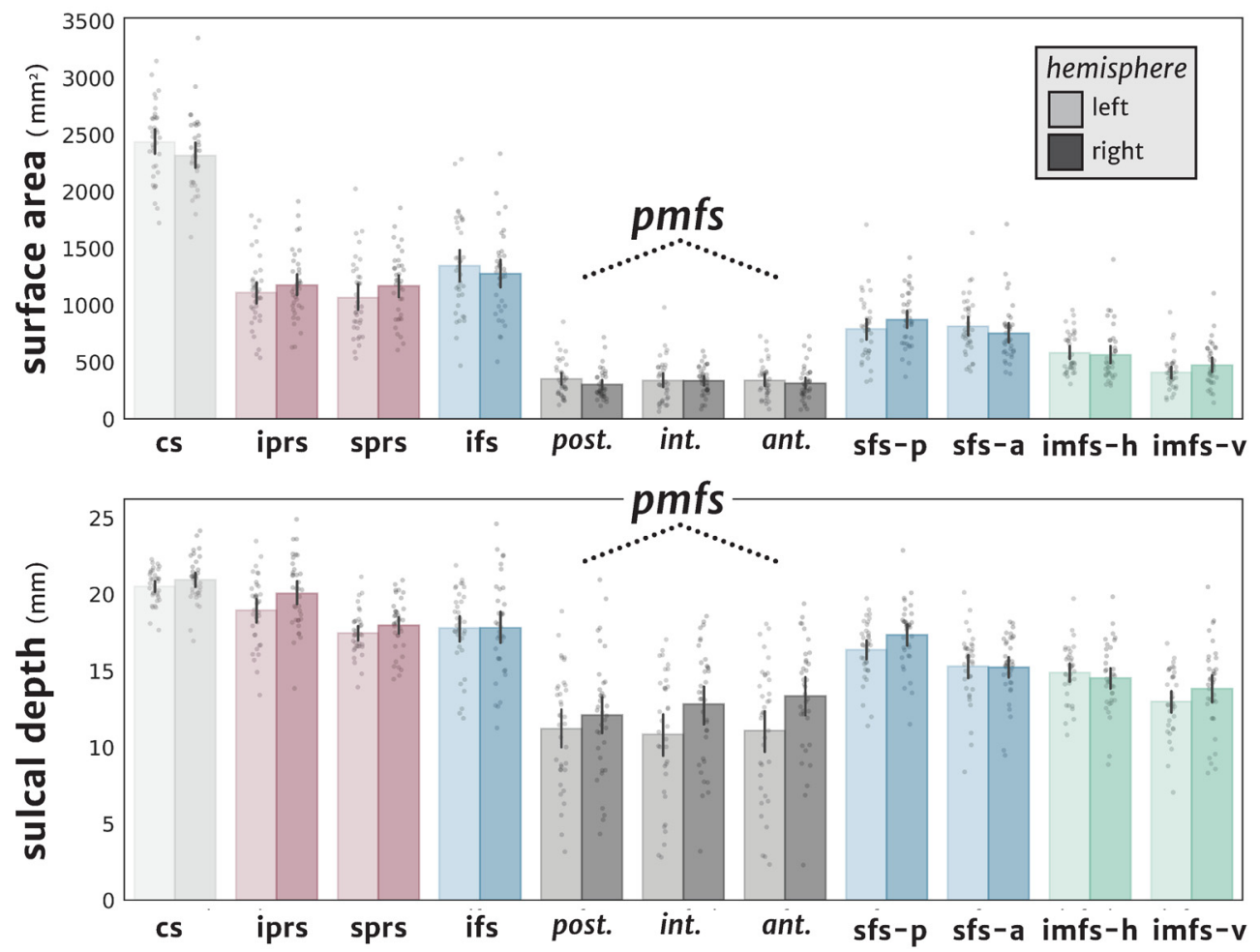

Figure 2. LPFC tertiary sulci are easily identifiable and characteristically shallow. $\boldsymbol{a}$, Left, An example inflated cortical surface of an individual left hemisphere in which the sulci examined in the present study are outlined and labeled (Extended Data Fig. 2-1 for all participants). Sulci are dark gray, while gyri are light gray. Right, Two postmortem hemispheres (Retzius, 1896) and three histological sections (note that the pmfs components are referred to as "intermediate frontal sulcus" in the Allen Human Brain Atlas: https://atlas.brain-map.org/; Ding et al., 2016) showing that the pmfs sulci are also identifiable in postmortem tissue samples. $\boldsymbol{b}$, Top, Surface area for each sulcus (ordered posterior to anterior) is plotted for each individual participant (gray circles), as well as the mean (colored bars) and 95\% confidence interval (black line). Acronyms used for each LPFC sulcus are also included. Darker shades indicate right hemisphere values, while lighter shades indicate left hemisphere values. The three pmfs sulci have the smallest surface area of all LPFC sulci measured in the present study. Bottom, Same layout as above, but for sulcal depth (in millimeters). The three pmfs sulci are the shallowest of the LPFC sulci measured here.

precentral sulcus (iprs). Superiorly, we identified the anterior sfs (sfs-a) and posterior sfs (sfs-p). Inferiorly, we identified the ifs. Anteriorly, we identified the imfs-h and imfs-v. The latter two sulci are consistent with the classic definition of the middle frontal sulcus by Eberstaller (1890), but have since been renamed (Fig. 1; Miller et al., 2020a). Within the posterior MFG, we identified three sulci in every hemisphere $(N=72)$. From posterior to anterior, the first sulcus (pmfs-p) is positioned immediately anterior to the sprs (Fig. 2a, Extended Data Fig. 2-1) and most commonly does not intersect other sulci (Table 1, summary of the morphological patterns or types). The second sulcus (pmfs-i) is located immediately anterior to the pmfs-p, and typically aligns with the separation between the sfs-a and sfs-p components. The pmfs- $i$ is most often independent [especially in the right 
Table 1. Most common intersections of the pmfs components (morphological types)

\begin{tabular}{llll}
\hline Most common intersections & 1st & 2nd & 3rd \\
\hline pmfs-p & Independent & pmfs-i & iprs \\
Ih & $44.4 \%$ & $22.2 \%$ & $16.7 \%$ \\
& Independent & sfs-a & pmfs-i \\
rh & $30.6 \%$ & $30.6 \%$ & $16.7 \%$ \\
& & & \\
pmfs-i & Independent & pmfs-p & pmfs-a \\
Ih & $47.2 \%$ & $22.2 \%$ & $16.7 \%$ \\
& pmfs-a & Independent & pmfs-p \\
rh & $58.3 \%$ & $27.8 \%$ & $19.4 \%$ \\
& & & \\
pmfs-a & Independent & imfs-h & pmfs-i \\
Ih & $47.2 \%$ & $38.9 \%$ & $16.7 \%$ \\
& imfs-h & pmfs-i & Independent \\
rh & $52.8 \%$ & $50.0 \%$ & $13.9 \%$ \\
\hline
\end{tabular}

hemisphere (rh)] or intersects [especially in the left hemisphere (lh)] the pmfs-a. Finally, the third sulcus (pmfs-a) is immediately anterior to the pmfs-i, inferior to the sfs-a, and posterior to the imfs-h. The pmfs-a most commonly intersects other sulci in the right hemisphere.

Each sulcus is also identifiable within individual in vivo volumetric slices (Petrides, 2019) and in postmortem brains (Fig. 2), which indicates that the computational process used to generate the cortical surface reconstruction in the MRI data does not artificially create these sulci within the MFG. Our results show that the pmfs is distinguishable from the imfs, which is in correspondence with the recent atlas from Petrides (2019), whereas the pmfs and imfs were often combined in classic sulcal atlases (Ono et al., 1990).

The two most identifying morphological features of the three pmfs sulci are their surface area and depth (Fig. 2b). Each pmfs sulcus is of approximately equal surface area (Fig. $2 b$, Table 2), which is smaller than the surface area of the other examined sulci in LPFC (Fig. 2b, Table 2). A two-way repeatedmeasures ANOVA with factors sulcus and hemisphere yielded a main effect of sulcus $\left(F_{(5.78,202.15)}=384.1, p<0.001, \eta_{G}^{2}=\right.$ $0.84)$ and no main effect of hemisphere $\left(F_{(1,35)}=0.1, p=0.77\right)$. The depth of the three pmfs sulci are also the shallowest of the lateral PFC sulci examined (Fig. 2b, Table 1). A two-way repeated-measures ANOVA with sulcus and hemisphere as factors yielded a main effect of sulcus $\left(F_{(3.15,103.84)}=77.7\right.$, $\left.p<0.001, \eta_{G}^{2}=0.55\right)$, and a main effect of hemisphere $\left(F_{(1,33)}=20.4, p<0.001, \eta_{G}^{2}=0.02\right)$ in which sulci were deeper in the right compared with the left hemisphere (Fig. $2 b$, Table $2)$. Post hoc tests show that, across hemispheres, the pmfs-p is shallower than all other sulci ( $p$ values $<0.001$, Tukey's adjustment), and the pmfs-i and pmfs-a are shallower than all other sulci except for the imfs-v. Together, three pmfs sulci are identifiable in individual hemispheres (Fig. 2, Extended Data Fig. 21) and distinguish themselves from other LPFC sulci based on their surface area and shallowness.

The pmfs-p, pmfs-i, and pmfs-a are anatomically dissociable and reflect a larger rostro-caudal myelination gradient in LPFC

While the pmfs-p, pmfs-i, and pmfs-a are morphologically distinct from surrounding sulci (Fig. 2), it is presently unknown whether they are anatomically and functionally similar or distinct from one another. To test this, we first extracted and compared average MRI $\mathrm{T}_{1} \mathrm{w} / \mathrm{T}_{2} \mathrm{w}$ ratio values from each sulcus. The
Table 2. Surface area and depth of the three pmfs components

\begin{tabular}{ccc}
\hline & Surface area $\left(\mathrm{mm}^{2}\right)$ & Depth $(\mathrm{mm})$ \\
\hline $\begin{array}{l}\text { pmfs-a } \\
\text { lh }\end{array}$ & \\
rh & $341.9 \pm 154.8$ & $11.1 \pm 4.4$ \\
pmfs-i & $315.4 \pm 149.7$ & $13.4 \pm 3.7$ \\
lh & & \\
rh & $339.3 \pm 191.7$ & $10.9 \pm 4.2$ \\
pmfs-p & $337.8 \pm 124.2$ & $12.8 \pm 3.8$ \\
h & & \\
rh & $353.6 \pm 164.1$ & $11.2 \pm 3.8$ \\
\hline Values are mean \pm SD. & $301.7 \pm 133.2$ & $12.1 \pm 3.9$ \\
\hline
\end{tabular}

$\mathrm{T}_{1} \mathrm{w} / \mathrm{T}_{2} \mathrm{w}$ ratio is a tissue contrast enhancement index that is correlated with myelin content (Fig. 3a; Glasser and Van Essen, 2011; Shams et al., 2019). We chose this index because myeloarchitecture is a classic criterion used to separate cortical areas from one another (Vogt and Vogt, 1919; Flechsig, 1920; Hopf, 1956; Dick et al., 2012). A two-way repeated-measures ANOVA with sulcus and hemisphere as factors yielded a main effect of sulcus $\left(F_{(1.76,61.7)}=85.0, p<0.001, \eta_{G}^{2}=0.39\right)$ and a main effect of hemisphere $\left(F_{(1,35)}=10.5, p=0.003, \eta_{G}^{2}=0.05\right)$ on myelin content, but no sulcus $\times$ hemisphere interaction $\left(F_{(1.73,60.5)}=2.5\right.$, $p=0.10)$. The differences in myelin across sulci were driven by the finding that $\mathrm{T}_{1} \mathrm{w} / \mathrm{T}_{2} \mathrm{w}$ ratio decreased from posterior to anterior across hemispheres [pmfs-p vs pmfs-i: $t_{(70)}=9.75, p<0.001$ (Tukey's post hoc); pmfs-i vs pmfs-a: $t_{(70)}=2.62, p=0.029$; pmfsp vs pmfs-a: $\left.t_{(70)}=12.37, p<0.001\right]$. The right hemisphere also had higher myelin content overall in the pmfs $\left(t_{(35)}=3.25\right.$, $p=0.003$ ). Accordingly, the three sulcal components are differentiable based on myelin content in both hemispheres (Fig. 3b).

The rostro-caudal gradient among the pmfs-p, pmfs-i, and pmfs-a sulci is embedded within a larger rostro-caudal myelination gradient in lateral PFC. Specifically, modeling $\mathrm{T}_{1} \mathrm{w} / \mathrm{T}_{2} \mathrm{~W}$ content across frontal sulci as a function of distance from the central sulcus (Fig. 3c) using a mixed linear model revealed a significant negative effect of distance from the central sulcus along the rostral-caudal axis $(\beta=-0.001, z=-33.8, p<$ $0.001)$, with no differences between hemispheres $(\beta=-0.003$, $z=-0.8, p=0.4$ ). Together, our quantifications show that the pmfs-p, pmfs-i, and pmfs-a are embedded within a larger anatomical and functional hierarchical gradient in LPFC (for further details, see Discussion).

The pmfs components show a microstructural profile across cortical layers that is distinct from the middle frontal gyrus Classic and modern findings show that there is generally more intracortical myelin in deeper cortical layers and that the depths of sulci often have less myelinated fibers than gyral crowns (Braitenberg, 1962; Sanides, 1972; Welker, 1990; Annese et al., 2004; Rowley et al., 2015). Building on this work, we sought to calculate microstructural profiles for myelin content across cortical depths for each pmfs component, as well as the gyral components of the MFG that surround them (Fig. 4; Materials and Methods). To do so, we implemented equivolume algorithms to construct cortical surfaces within the gray matter. The depth profiles from equivolume surfaces have been used to investigate cortical laminar organization in vivo and to correspond with those obtained from both ex vivo MRI data and postmortem histological sections (Waehnert et al., 2014; Paquola et al., 2019).

The MFG and pmfs components show distinct microstructural profiles of myelin content across cortical depths. A three- 

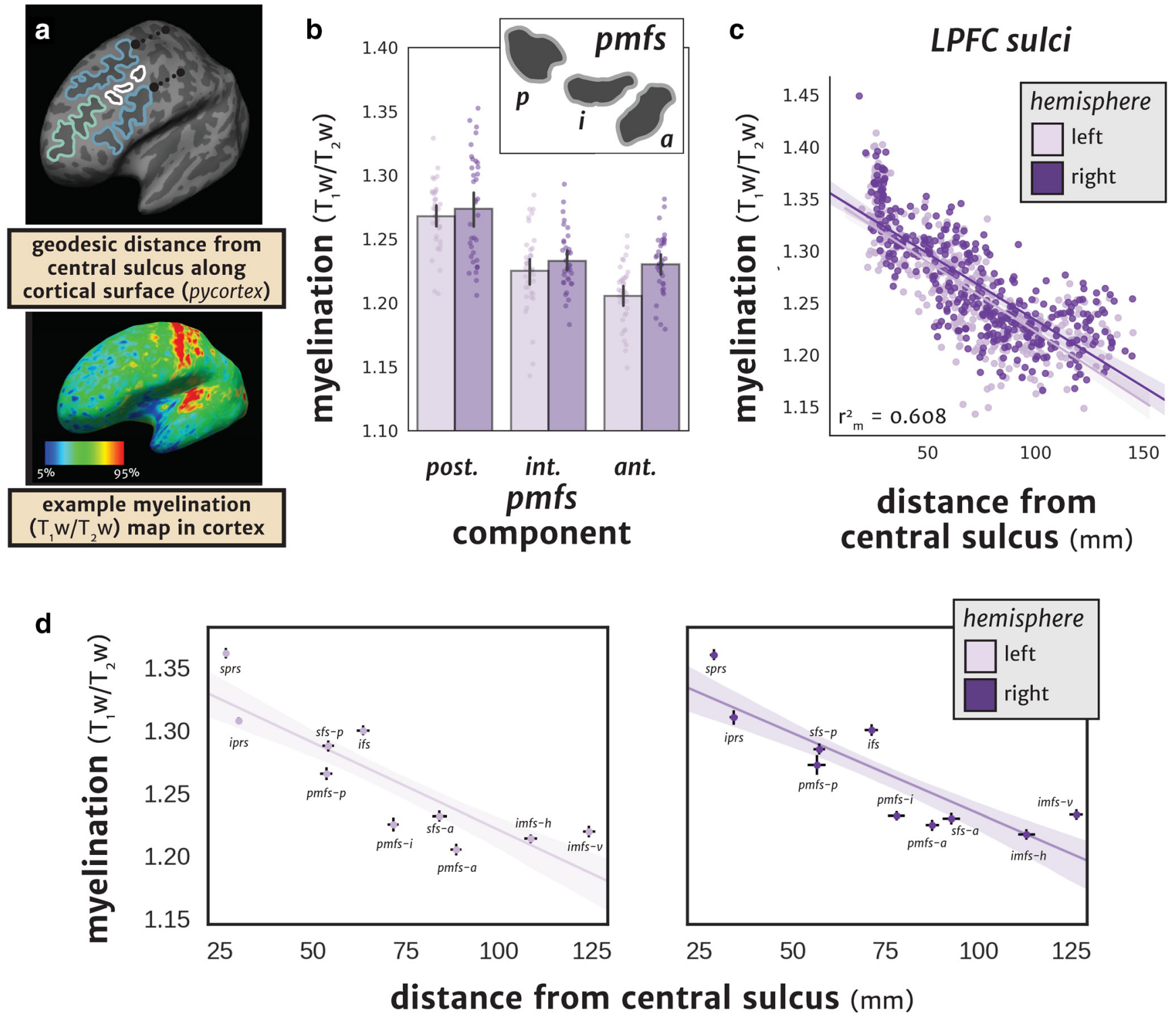

Figure 3. The pmfs sulci are anatomically differentiable based on myelin content. $\boldsymbol{a}$, Top, Schematic of the calculation of geodesic distance along the cortical surface. For each sulcus, the average distance of each vertex from the central sulcus was calculated (dotted black line; Materials and Methods). Bottom, An example $\mathrm{T}_{1} \mathrm{w} / \mathrm{T}_{2} \mathrm{w}$ map in an individual participant in which the $5-$ $95 \%$ percentiles of values are depicted. $\boldsymbol{b}, \mathrm{T}_{1} \mathrm{w} / \mathrm{T}_{2} \mathrm{w}$ values (a proxy for myelin content) are plotted for each component of the pmfs for each individual participant $(N=36)$. Bars represent the mean $\pm 95 \% \mathrm{Cl}$, while each participant is depicted as a circle. Darker shades indicate right hemisphere values, while lighter shades indicate left hemisphere values. The components of the pmfs are differentiable based on myelin content, with a decrease from posterior to anterior across both hemispheres. $\boldsymbol{c}$, Scatterplot showing the negative relationship between distance from the central sulcus and the mean myelination value for all labeled sulci from each individual ( $N=36$ participants). The mixed linear model (Materials and Methods) with predictors of distance and hemisphere shows a marginal $r^{2}$ of $60.8 \%$. Scatterplot is bootstrapped at $68 \% \mathrm{Cl}$ for visualization. $\boldsymbol{d}$, Scatterplot showing the mean $\mathrm{T}_{1} \mathrm{w} / \mathrm{T}_{2} \mathrm{~W}$ value for each sulcus as a function of distance (in millimeters) from the central sulcus. Error bars for both the $x$ - and $y$-axes represent SEM $(68 \% \mathrm{Cl})$ across individuals $(N=36$ participants). Dark purple, right hemisphere; light purple, left hemisphere.

way repeated-measures ANOVA with factors of structure (pmfsp, pmfs-i, pmfs-a, MFG), cortical depth (0\%, 12.5\%, 25\%, 37.5\%, $50 \%, 62.5 \%, 75 \%, 87.5 \%, 100 \%)$, and hemisphere (left, right) yields main effects of structure $\left(F_{(2.26,78.94)}=15.6, p<0.001, \eta_{G}^{2}=\right.$ $0.007)$, depth $\left(F_{(1.39,48.49)}=1849.6, p<0.001, \eta_{G}^{2}=0.84\right)$, and a structure $\times$ depth interaction $\left(F_{(6.78,237.43)}=78.5, p<0.001, \eta_{G}^{2}=\right.$ $0.02)$. This interaction between structure and depth did not differ by hemisphere $\left(F_{(4.69,164.26)}=1.13, p=0.35, \eta_{G}^{2}=0.02\right)$, so subsequent analyses are collapsed across hemispheres. To determine which differences drive the distinct profiles in myelin content across cortical layers between the pmfs and MFG, we conducted post hoc tests at each cortical depth (Fig. 4a). The MFG had higher myelin content in each of the upper cortical depths $(0 \%$, $12.5 \%, 25 \%, 37.5 \%)$ compared with all of the pmfs components (all $p$ values $<0.001$, FWE corrected at $\alpha=0.05 / 9$ for the nine cortical depths). In the middle-to-deep layers (50\%, 62.5\%), the pmfs-p had higher myelin content than either the pmfs-i $(50 \%$ : $\left.t_{(105)}=6.4, p<0.001 ; 62.5 \%: t_{(105)}=7.0, p<0.001\right)$ or pmfs-a (50\%: $\left.t_{(105)}=7.1, p<0.001 ; 62.5 \%: t_{(105)}=8.1, p<0.001\right)$, and was even higher than the MFG (50\%: $t_{(105)}=0.27, p=0.99$; 62.5\%: $\left.t_{(105)}=3.7, p=0.002\right)$. At the deepest cortical layers, closest to the gray/white matter boundary, all three pmfs components showed increased myelin relative to the MFG. Specifically, the pmfs-a showed the highest myelin content in the deepest layers, but all three pmfs components displayed higher myelin than the MFG (all $p$ values $<0.001$, FWE corrected at $\alpha=0.05 / 9$ for the nine cortical depths). The profile of myelin content across cortical depths in the pmfs and MFG is also robust when 

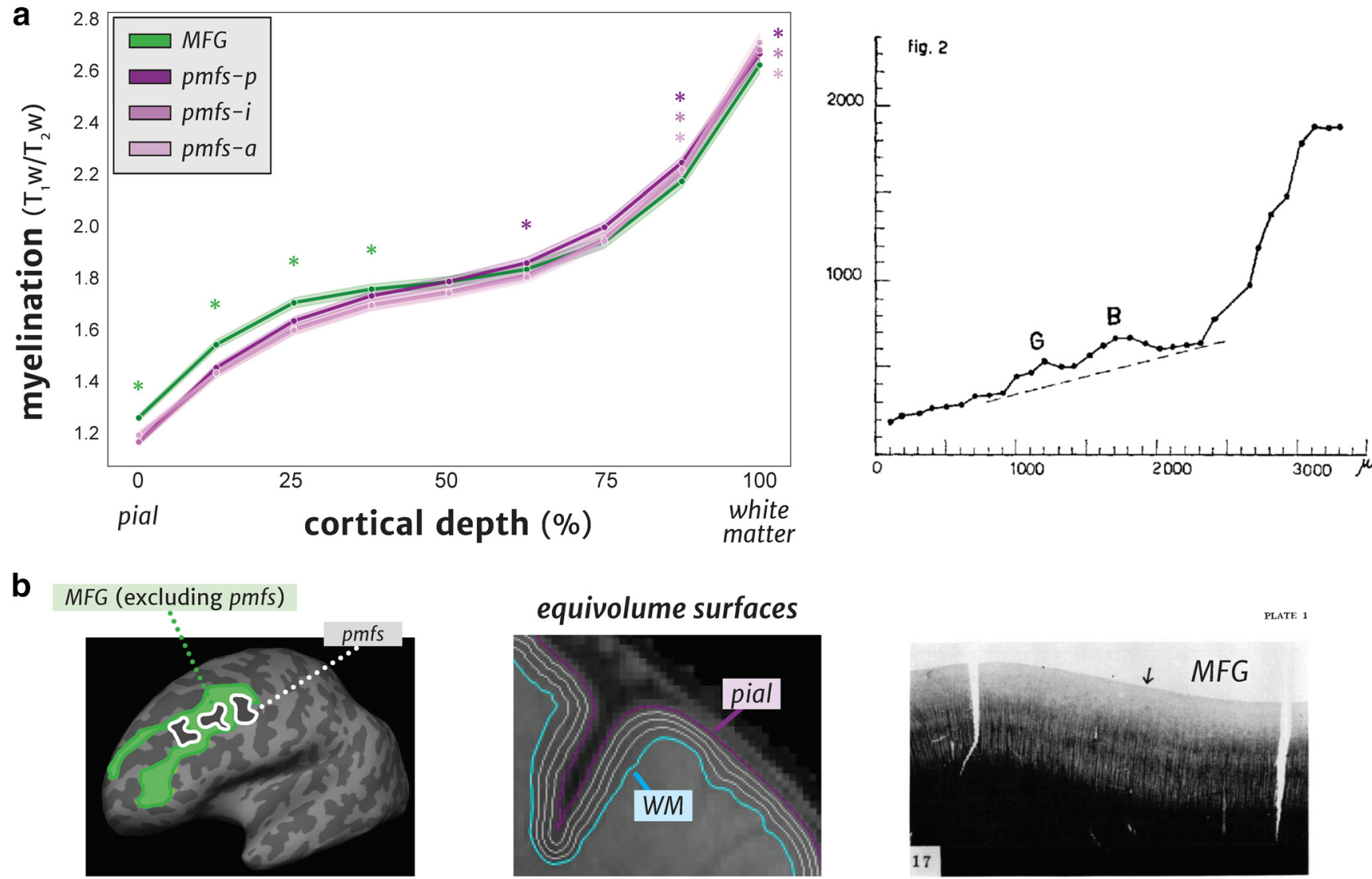

Figure 4. The pmfs sulci and middle frontal gyrus have differentiable myelin profiles across cortical depths. $\boldsymbol{a}$, Left, Tissue contrast enhancement $\left(T_{1} w / T_{2} w\right.$ metric, a proxy for myelin) at nine cortical depths, sampled from the outer gray matter (pial) to the gray/white matter boundary (white matter) using equivolume surfaces (Materials and Methods). The MFG (excluding the pmfs) has higher myelin content than all pmfs components in the upper cortical layers, while the pmfs components have higher myelin content in deeper layers. Shaded area represents bootstrapped $68 \% \mathrm{Cl}$ across participants. Green asterisks show significant statistical differences between the MFG and all pmfs components (MFG $>$ pmfs), while purple asterisks show the reverse (pmfs > MFG; all tests FWE corrected at $p<0.05 / 9)$. Right, Myelinated fiber density $(y$-axis) profile across cortical depths $(x$-axis) in postmortem histological sections of the MFG, adapted from Braitenberg (1962). B, Stria of Baillarger; G, Stria of Gennari. Similar to our measurements, myelination increases from outer to inner layers within the MFG (reproduced with copyright permission). $\boldsymbol{b}$, Left, Individual left hemisphere with the manually defined pmfs components (white) and the surrounding MFG (green) as defined by FreeSurfer (Destrieux et al., 2010). We excluded the pmfs components from the MFG to test for anatomically distinct profiles. Middle, Example equivolume surfaces at five different cortical depths, from the pial to white matter surfaces, which were used to sample the $\mathrm{T}_{1} \mathrm{w} / \mathrm{T}_{2} \mathrm{w}$ metric across depths. Right, Myelination stain of a postmortem histological section of the MFG from Braitenberg (1962). Arrow, Location from which the myelinated fiber density profile (a, right) was calculated (reproduced with copyright permission).

comparing myelin content at a coarser (three instead of nine) level of upper, middle, and lower depths (mean of depths within each bin): structure $\times$ depth interaction $\left(F_{(3.87,135.4)}=127.4, p<0.001, \eta_{G}^{2}=0.02\right)$. Altogether, the pmfs differed from the MFG in microstructure across cortical layers, with lower myelin content in upper layers and higher myelin content in deeper layers. This surface-based sampling of cortical depths provides in vivo neuroimaging evidence for a microanatomical distinction of the pmfs from the surrounding MFG. Further, the depth profiles of $\mathrm{T}_{1} \mathrm{w} /$ $\mathrm{T}_{2} \mathrm{~W}$ values within the MFG are similar to classic myeloarchitectural quantifications of the MFG (Fig. 4).

The pmfs-p, pmfs-i, and pmfs-a exhibit different characteristic patterns of whole-brain functional connectivity

To determine whether the pmfs-p, pmfs-i, and pmfs-a are functionally distinct, we leveraged detailed individual functional parcellations of the entire cerebral cortex based on functional connectivity from a recently published study (Kong et al., 2018; Fig. 5a). Importantly, this parcellation was conducted blind to both cortical folding and our sulcal definitions. Within each hemisphere in the same participants in which we generated manual sulcal labels, we generated a functional connectivity network profile (which we refer to as a "connectivity fingerprint"). For each sulcal component, we calculated the overlap between 17 functional networks (on the native hemisphere, based on the Dice coefficient; Materials and Methods). This technique generated a cortical topography reflective of the whole-brain connectivity patterns for each sulcal component (Fig. $5 a$, bottom), and can be interpreted, similar to other studies of functional network variations (Gordon et al., 2017; Seitzman et al., 2019), as a traitlike connectivity profile for each pmfs component within each participant.

Our approach demonstrated that the pmfs-p, pmfs-i, and pmfsa have different connectivity fingerprints and thus, are functionally dissociable. Average connectivity fingerprints across participants are illustrated in Figure 5b. A repeated-measures ANOVA with sulcal component (pmfs-p, pmfs-i, pmfs-a), hemisphere (left, right), and network yielded a significant component $\times$ network interaction $\left(F_{(32,1120)}=45.2, p<0.001, \eta_{G}^{2}=0.29\right)$, as well as a component $\times$ network $\times$ hemisphere interaction $\left(F_{(32,1120)}=5.26, p<0.001\right.$, $\eta_{G}^{2}=0.040$; Fig. 5b). In each hemisphere, there is a component $\times$ network interaction (left: $F_{(32,1120)}=29.4, p<0.001, \eta_{G}^{2}=0.35$; right: $\left.F_{(32,1120)}=23.2, p<0.001, \quad \eta_{G}^{2}=0.27\right)$ in which the difference between hemispheres is driven by the pmfs-p connectivity 


\section{a group network parcellation}

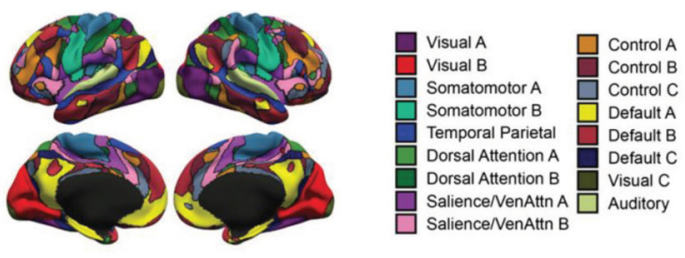

17-network resting-state functional connectivity profiles in each indvidual participant

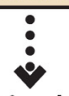

individual cortical topographies
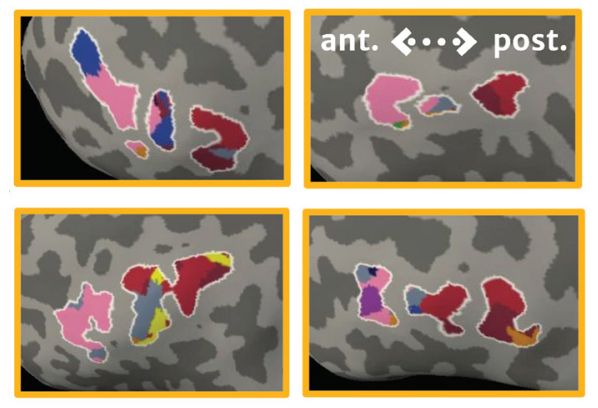

calculate overlap between connectivity network profile and pmfs component in each individual

\section{b pmfs connectivity fingerprints (mean across participants)}

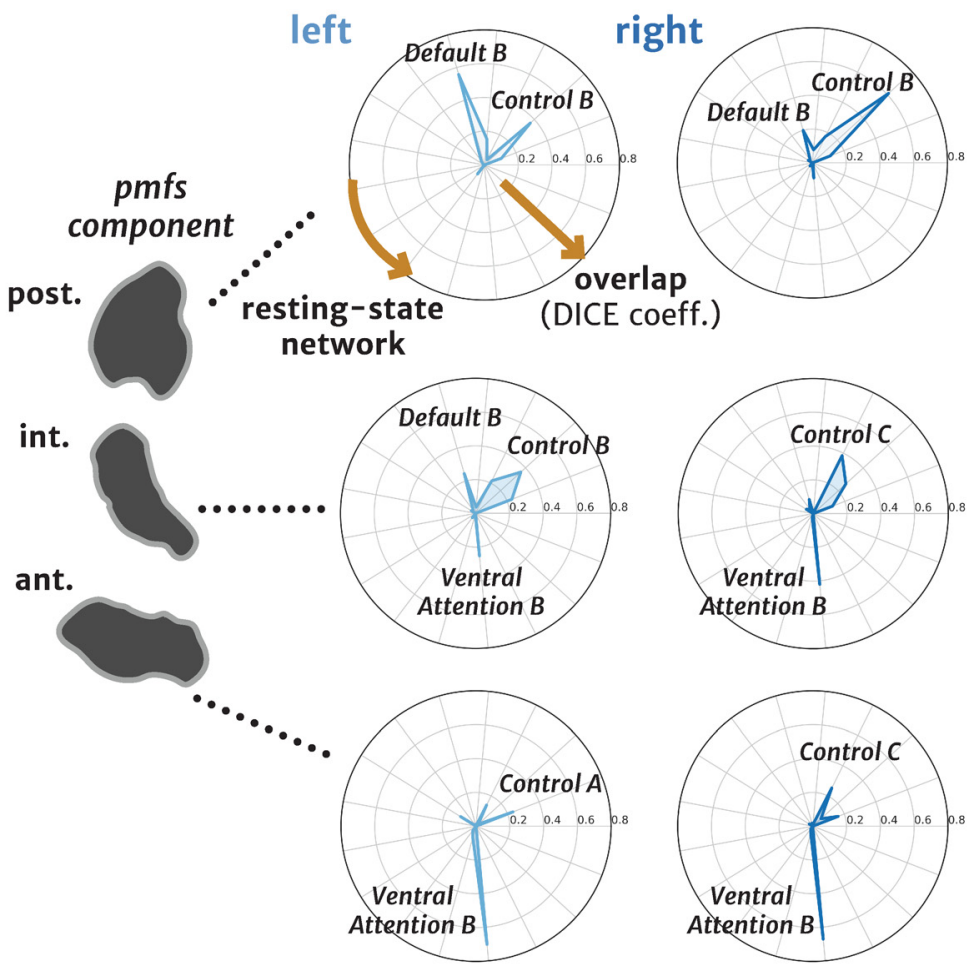

\section{C individual connectivity fingerprints}

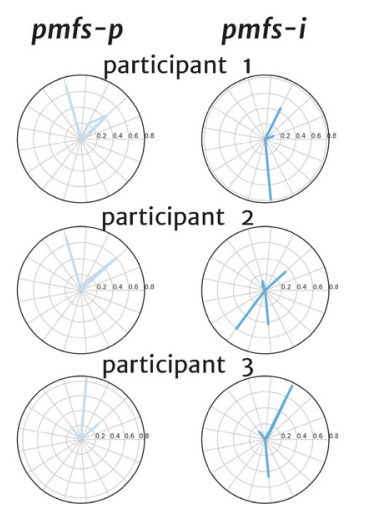

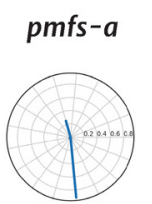
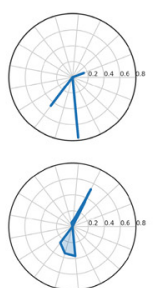
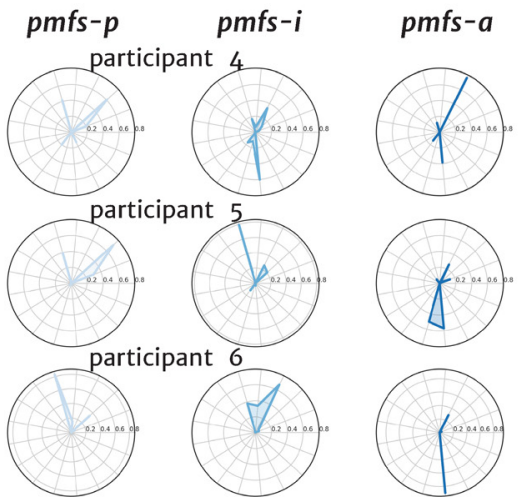

variability across participants

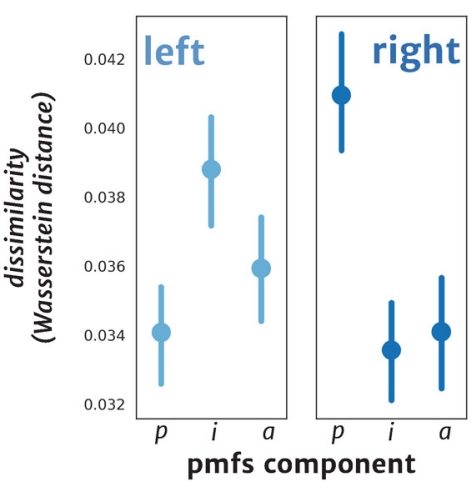

Figure 5. The pmfs components are functionally differentiable based on connectivity fingerprints within individuals. $\boldsymbol{a}$, Schematic of how individual-level resting-state connectivity profiles were generated in each participant. Resting-state network parcellations for each participant were obtained from a recent study (Kong et al., 2018) in an observer-independent fashion of sulcal definitions in LPFC (reproduced with copyright permission). Example individual cortical topographies are shown in four individual participants, colored according to the group parcellation. The individual cortical topographies and pmfs sulcal definitions were used to calculate the connectivity fingerprint, which represents the overlap of each network within the pmfs component of each participant. $\boldsymbol{b}$, Polar plots showing the mean connectivity fingerprint of the three pmfs components (plotted outward) with each of 17 resting-state functional connectivity networks, across participants. Resting-state networks with the highest overlap across participants are labeled. $c$, Left, Polar plots showing variability among six individual participants. Right, Dissimilarity of the resting-state network fingerprints (variability in the connectivity fingerprint across participants represented by the Wasserstein distance between unique pairs of participants; Materials and Methods) are plotted as a function of each pmfs component for left and right hemispheres. Error bars represent $68 \%$ Cl (SEM) across unique participant pairs.

fingerprint. Specifically, the pmfs-p overlaps most with the default mode network in the left hemisphere and the cognitive control network in the right hemisphere.

Additionally, there are also individual and hemispheric differences in the connectivity fingerprint of each pmfs component at the level of individual participants (Fig. $5 c$; Extended Data Fig. 51). To characterize individual differences, we built on work showing network connectivity variations across individuals (Kong et al., 2018; Seitzman et al., 2019) by relating this connectivity variability to individual anatomical landmarks in LPFC.
We quantified connectivity fingerprint variability by measuring the pairwise Wasserstein distance between the connectivity profiles for all unique participant pairs for each sulcal component, in which a larger distance indicates decreased similarity, and therefore greater variability (see Materials and Methods). This approach quantifies how variable the pattern of network overlap (connectivity fingerprint) is across individuals for each pmfs component (Fig. $5 c$, right). In the right hemisphere, the pmfs-p showed the most variable network profile across all unique participant pairs (pmfs-p vs pmfs-i: Wilcoxon signed- 


\section{a generating meta-analytic sulcal-functional mappings}

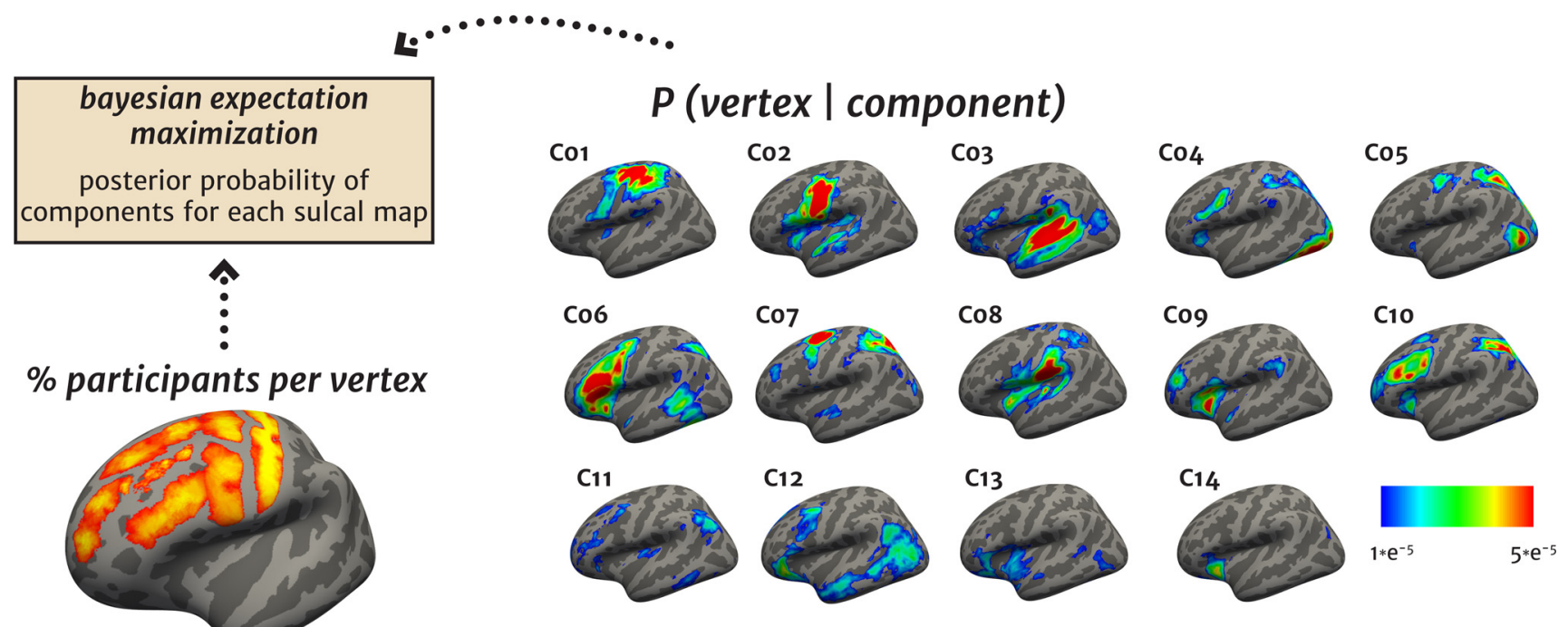

\section{sulcal maximum probability maps}

cognitive component maps

b

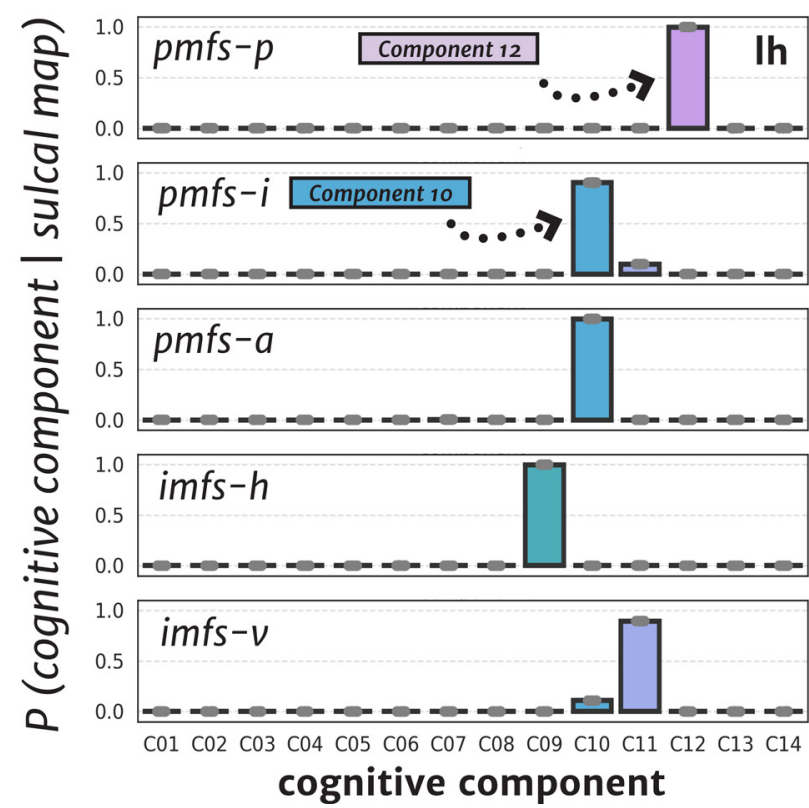

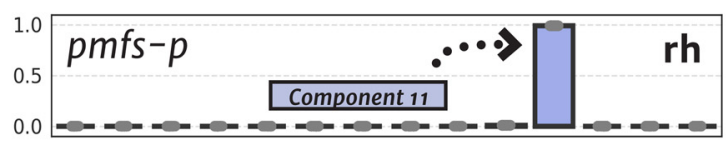
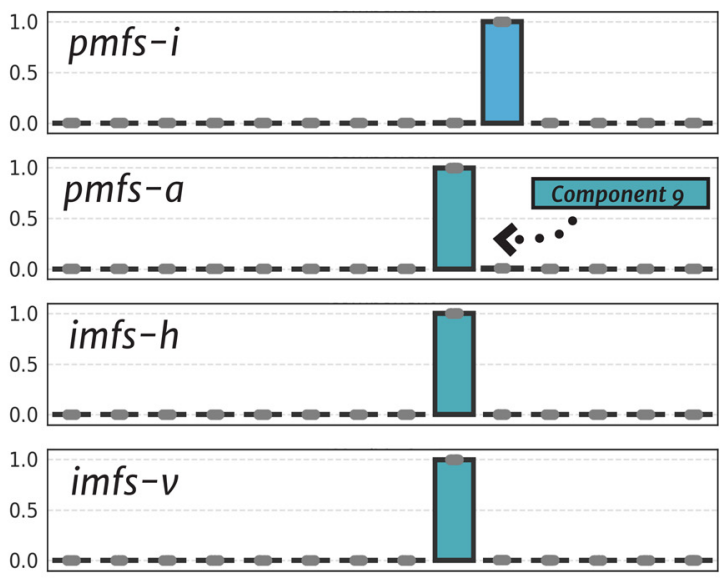

C01 C02 C03 C04 C05 C06 C07 C08 C09 C10 C11 C12 C13 C14

cognitive component

Figure 6. The pmfs and imfs components are functionally differentiable based on cognitive components: a meta-analysis of fMRl experimental tasks. $\boldsymbol{a}$, Schematic of analyses linking sulcal probability maps (bottom, left) and cognitive component maps (right) from a meta-analysis of fMRI experimental tasks (Yeo et al., 2015) using an expectation maximization algorithm (Materials and Methods). For each pmfs component, the algorithm provides a posterior probability for each of 14 cognitive components being associated with the provided sulcal probability map. $\boldsymbol{b}$, For each pmfs and imfs component in each hemisphere, the posterior probability for each cognitive component is plotted. This approach further supports that the pmfs-p (component 12, Ih; component 11, rh), pmfs-i (component 10, lh and rh), and pmfs-a (component 10, Ih; component 9, rh; Materials and Methods) are functionally dissociable based on meta-analytic data of cognitive task activations. The imfs-h and imfs-v are also dissociable from the pmfs components in the left hemisphere, and are functionally similar to the pmfs-a in the right hemisphere. Gray dots indicate individual participant data points when the analysis is performed with individual labels transformed to a template cortical surface rather than with probability maps (Materials and Methods).

rank test $(W)=7.2 \times 10^{4}, \quad p<0.001 ;$ pmfs-p vs pmfs-a: $W=7.4 \times 10^{4}, p<0.001$ ), while the pmfs-i was most variable in the left hemisphere (pmfs-i vs pmfs-a: $W=8.8 \times 10^{4}$, $p=0.014$; pmfs-i vs pmfs-p: $\left.W=8.0 \times 10^{4}, p<0.001\right)$. This analysis suggests that the right pmfs-p and left pmfs-i mark regions of LPFC with particularly high levels of individual differences in functional connectivity profiles, providing an anatomical substrate for network connectivity differences across individuals.

The pmfs-p, pmfs-i, and pmfs-a are functionally dissociable: meta-analyses across 83 experimental task categories We next tested whether the dissociation of functional networks among the pmfs-p, pmfs-i, and pmfs-a identified in individual 


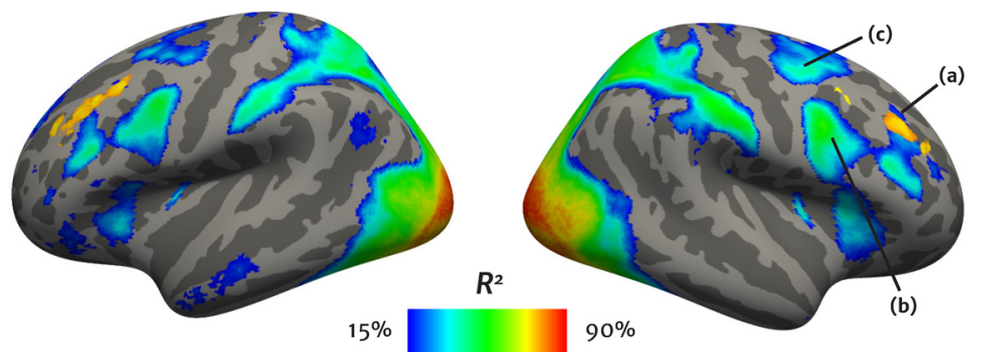

Figure 7. Comparing the overlap between retinotopic responses relative to the predicted location of the pmfs sulcal components. Map of the mean $(n=181) R^{2}$ metric (colorbar) from the HCP retinotopy dataset (Benson et al., 2018) on the fsaverage template cortical surface for each hemisphere, thresholded at $15 \%$. This metric measures how well the fMRI time series at each vertex is modeled by population receptive field (pRF) modeling that was calculated and shared by Benson et al. (2018; https://osf.io/bw9ec/wiki/home/). Predicted pmfs location from the maximum probability maps is overlaid in orange (thresholded at $33 \%$ overlap across participants). $\boldsymbol{a}$, There was only a modest overlap between predicted pmfs location and retinotopic representations in the right hemisphere (no overlap in the left hemisphere). $\boldsymbol{b}, \boldsymbol{c}$, Instead, and consistent with prior work (Mackey et al., 2017), the highest correspondence between retinotopic responses and sulcal patterning in LPFC occurs at the following two sulcal intersections: (1) the sprs and sfs-p (c); and (2) the iprs and ifs $(\boldsymbol{b})$.

participants (Fig. 5) can also be observed in meta-analytic analyses of functional activation data at the group level. That is, do the components of the pmfs show a functional dissociation of engagement over a wide array of cognitive operations? To test for different patterns of functional activations across tasks, we generated sulcal probability maps on a template cortical surface (Fig. 6a, bottom left). Analogous to probabilistic maps for functional regions (Wang et al., 2015; Weiner et al., 2017, 2018), the maps provide a vertex-wise measure of anatomical overlap across individuals for all 13 LPFC sulci examined in the present study. As the pmfs components disappear on average templates (Fig. 1 ), these probabilistic maps are independent of the sulcal patterning of the template itself, which merely serves as a cortical surface independent of each individual cortical surface. We then compared these sulcal probability maps to 14 probabilistic "cognitive component" maps derived from an author-topic model of meta-analytic activation data across 83 experimental task categories (Yeo et al., 2015).

The cognitive component model links patterns of brain activity to behavioral tasks via latent components representing putative functional subsystems (Yeo et al., 2015). Each cognitive component map (which was calculated on the same template cortical surface used here) provides the probability that a given voxel will be activated by each of the 14 components (across all 83 tasks). We then used an expectation maximization algorithm (via posterior probability; Materials and Methods) to relate brain activity in each sulcal probability map to each cognitive component (Fig. $6 a$, right). Importantly, when calculating the posterior probabilities, we implemented a leave-one-participant-out crossvalidation procedure when constructing the sulcal probability maps to assess variability in the generated posterior probabilities for each cognitive component (Fig. 6b). To indicate the feasibility of this approach, the somatomotor components of the cognitive component map [component 1 (C01), C02] align most highly with the central sulcus as one would expect, which shows the ability of this method to measure structural-functional correspondences at the meta-analytic level.

This approach further reveals that the pmfs-p, pmfs-i, and pmfs-a are functionally dissociable based on meta-analytic data of cognitive task activations. In the right hemisphere, the pmfs-p, pmfs-i, and pmfs-a showed distinct probabilities for separate cognitive components: (1) the pmfs-p loaded onto a default mode component (C11); (2) the pmfs-i loaded onto an executive function component (C10); and (3) the pmfs-a loaded onto an inhibitory control component (C09). In the left hemisphere, the pmfs-a and pmfs-i both loaded onto an executive function (C10) component, while the pmfs-p loaded onto an emotional processing/episodic memory component (C12). The pmfs was also dissociable in activation profiles from the more anterior imfs. In the left hemisphere, the imfs showed no overlap with the pmfs, with the imfs-h loading onto the inhibitory control component (C09) and the imfs-v loading onto a default mode component (C11). In the right hemisphere, both the imfs- $h$ and imfs- $v$ loaded onto the same inhibitory control component (C09) as the pmfs-a.

Like our individual participant analyses, there were also hemispheric differences: the cognitive components overlapping the most with the pmfs-a and pmfs-p differed between the two hemispheres. The pmfs-p loaded onto an emotional processing/episodic memory component in the left hemisphere (Fig. 6b, top row) and a default mode component in the right hemisphere (Fig. $6 b$, top row), while the pmfs-a loaded onto an executive function component in the left hemisphere (Fig. 6b, third row) and an inhibitory control component in the right hemisphere (Fig. $6 b$, third row).

Finally, previous studies have identified retinotopic representations in human LPFC (Hagler and Sereno, 2006; Kastner et al., 2007; Mackey et al., 2017), but the three pmfs components did not overlap with cognitive components associated with visual processing in these meta-analytic analyses. To further examine the relationship between the pmfs components and visual processing, we analyzed whether the pmfs components explained a significant amount of variance (Fig. 7) in a newly published, whole-brain dataset of population receptive field measurements in 181 participants (Benson et al., 2018). When considering voxels that demonstrate retinotopic responses $\left(R^{2}>15 \%\right)$, the highest overlap between predicted pmfs location and retinotopic representations was specific to the right hemisphere for the pmfs-i (mean $R^{2}$ across participants $=28.5 \%$ ), with less overlap in the left hemisphere (all other pmfs $R^{2}$ values $<20 \%$ ). The most consistent correspondence between visual field maps and sulcal location occurred at (1) the intersection of the sprs and sfs-p, and (2) the intersection of the iprs and ifs, as previously reported (Mackey et al., 2017; Fig. 7). The iprs showed the highest retinotopic responses of the LPFC sulci (lh, 34.2\%; rh, 48.9\%) measured here, and this is also consistent with a recent study identifying a region critical for conditional eye movements within a similar location in the ifs (Germann and Petrides, 2020). Future studies examining the relationship between pmfs components and retinotopic representations in individual participants will further expand on these findings.

\section{Extensive individual differences in the location of the pmfs across individuals}

Although the three pmfs components are prominent within each hemisphere, there is extensive individual variability in the precise location of each sulcal component within the posterior MFG. To determine how well the probability maps could predict the location of the pmfs-p, pmfs-i, and pmfs-a within individual hemispheres, we used a cross-validated approach, iteratively leaving 


\section{a quantifying sulcal location with probability maps}
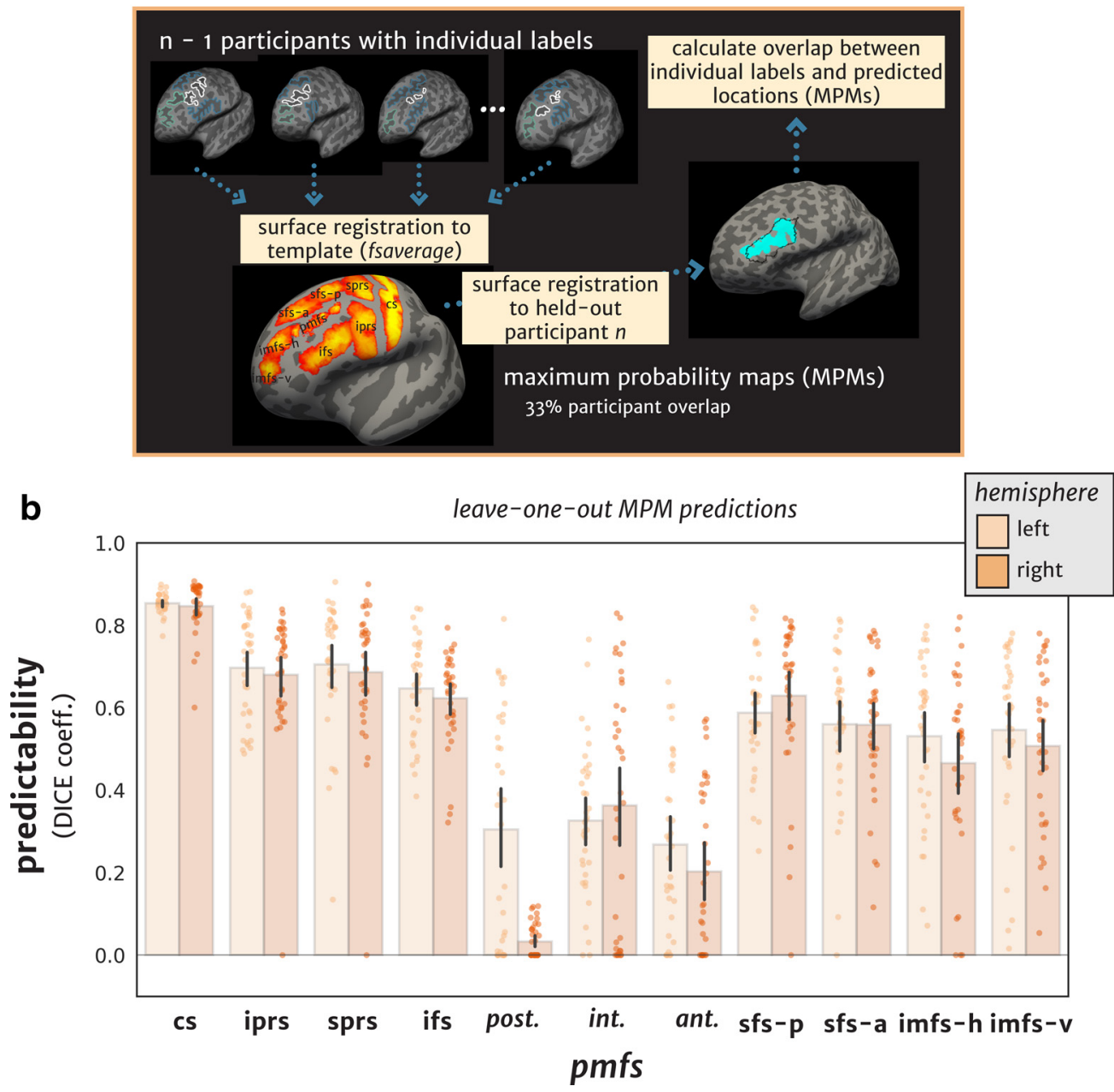

Figure 8. Quantification and prediction of pmfs-p, pmfs-i, and pmfs-a within individual hemispheres. $\boldsymbol{a}$, Procedure to generate sulcal probability maps based on the manual anatomical labeling within each individual participant. Labels from each individual are transformed to a template cortical surface to form a probabilistic sulcal map and then projected onto the surface of a held-out individual participant. The overlap between the manual anatomical label on the held-out participant and predicted location was then calculated for each iteration across participants. $\boldsymbol{b}$, Overlap (Dice coefficient) between predicted and manual location of each pmfs component within individual participants. Prediction for the pmfs is highest when all three components are combined. The cs is included as a noise ceiling for reference, as this landmark is used in the surface registration algorithm that aligns cortical surfaces across participants.

out one participant from the calculation of probability maps (Fig. 8a). Then, the MPMs were projected to the held-out individual's native cortical surface to calculate the overlap between the manually identified and probabilistically identified sulcal locations. This procedure resulted in a measure of location variability for each sulcal component (Fig. 8b). For these calculations, we used the cs as a noise ceiling (left, cs $=0.85 \pm 0.02$; right, $c s=0.85 \pm 0.06)$ as it is (1) considered very stable across individuals (see Materials and Methods) and (2) used in the cortex-based alignment procedure (Fischl et al., 1999b).

The pmfs components exhibited significant variability in sulcal location across participants (left: pmfs-p $=0.30 \pm 0.28$; pmfs$\mathrm{i}=0.32 \pm 0.18$; pmfs-a $=0.27 \pm 0.20$; right: pmfs-p $=0.03 \pm 0.04$; pmfs-i $=0.37 \pm 0.18$; pmfs-a $=0.20 \pm 0.20$ ). A two-way repeatedmeasures ANOVA with pmfs sulcal component (pmfs-p, pmfs-i, pmfs-a) and hemisphere (right, left) revealed a sulcus $\times$ hemisphere interaction $\left(F_{(1.84,64.47)}=9.52, p<0.001, \eta_{G}^{2}=0.08\right)$ driven by the finding that the pmfs-p is highly variable across individuals, resulting in very little predictability in the right hemisphere (Fig. 8b). When using all three pmfs components together, prediction is more robust (left, $\mathrm{pmfs}=0.41 \pm 0.13$; right, pmfs $=0.37 \pm 0.15$ ), but still is much lower than the predictability of the cs and also lower than prediction performance for all other LPFC sulci quantified in the present study (Fig. 8b). These results demonstrate that although the pmfs is prominent within each individual (Extended Data Fig. 2-1), the location of each pmfs component is variable across individuals, which provides empirical support for the historical confusion regarding its identification and labeling (Fig. 1).

\section{Discussion}

Here, we examined the relationship between cortical anatomy and function in human LPFC and showed for the first time (to our knowledge) that the pmfs serves as a meso-scale link between myelin content and functional connectivity in individual participants. The pmfs is a characteristically shallow tertiary sulcus with three components that differ in their myelin content, resting-state connectivity profiles, and engagement across meta-analyses of 83 cognitive tasks. We first discuss how these findings suggest modern empirical support for a classic, yet largely unconsidered, 
a
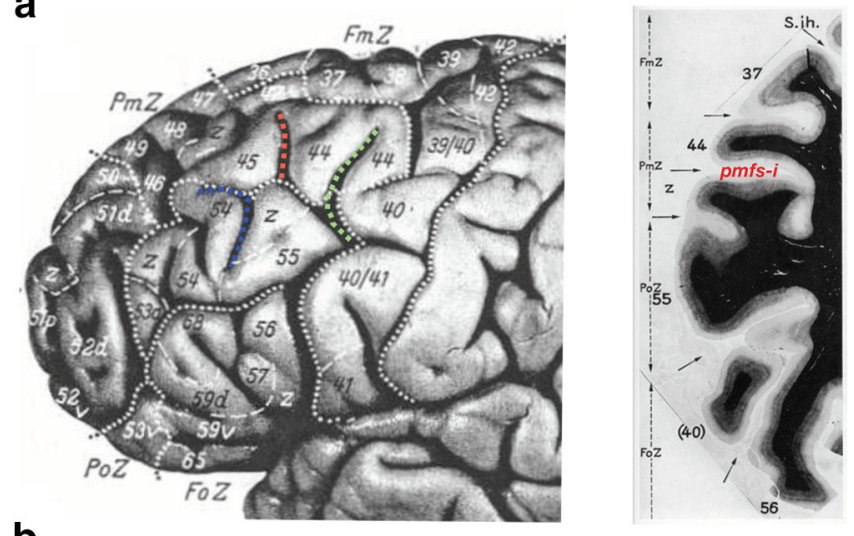

b

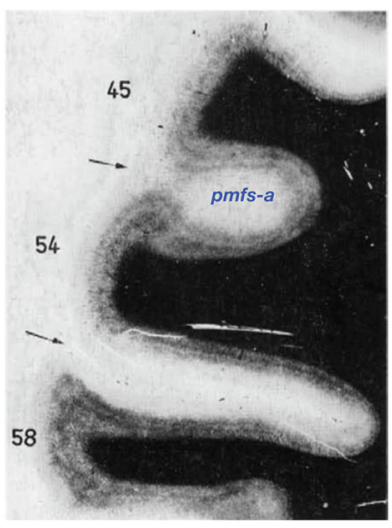

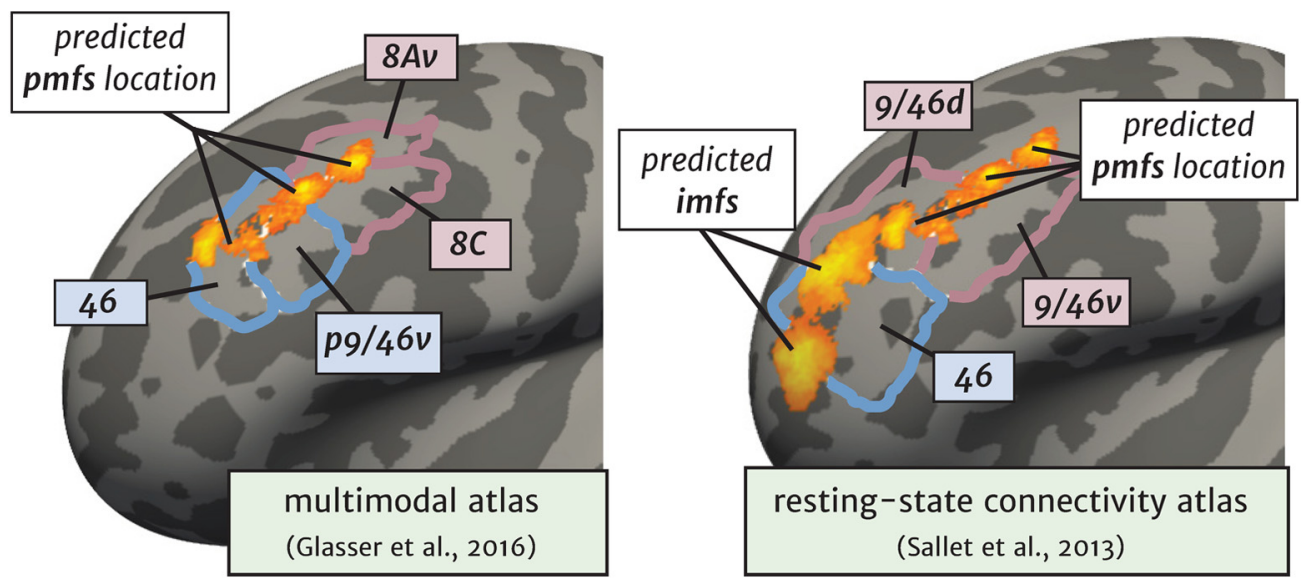

Figure 9. Linking the past to the present: myelination gradients, cortical areas, and the pmfs. $\boldsymbol{a}$, Left, Photograph of a left hemisphere from the study by Sanides (1962) (reproduced with copyright permission). Numbers indicate cortical areas differing in myeloarchitecture. Dotted white lines, sulcal boundaries as defined by Sanides (1962). Dotted colored lines (based on modern definitions used in the present study): pmfs-p, green; pmfs-i, red; and pmfs-a, blue. Identifying pmfs components in the classic images by Sanides (1962) shows that he identified myeloarchitectonic gradations within pmfs components, which is consistent with the present measurements. Gradations occurred in superior-inferior as well as anterior-posterior dimensions. In the inferior portion of the pmfs-p (green), there is an anterior-posterior transition between areas 40 and 55 . In the pmfs-i (red), there are two transitions: (1) a superior-inferior transition between areas 44 and a transition zone to area 55; and (2) an anterior-posterior transition between areas 44 and 45 . In the pmfs-a, there is a transition between areas 45 and 54 . Right, Myelination stain of a histological section (coronal orientation) from Sanides (1962) (reproduced with copyright permission). Arrows indicate boundaries between labeled myeloarchitectonic areas (numbers). pmfs-a is labeled to help the reader link the myelination stain to the image at left. The reader can appreciate the shallowness of the pmfs-a relative to the sulcus (ifs) between areas 54 and 58 , which is also consistent with our measurements (Fig. 2). $\boldsymbol{b}$, Left, Maximum probability maps (thresholded at 33\% overlap across participants) for the pmfs-p, pmfs-i, and pmfs-a are shown on the FreeSurfer average template (left hemisphere). The probability maps are shown relative to four areas from a multimodal cortical parcellation based on structural and functional MRI data (Glasser et al., 2016). The pmfsa appears to denote the dorsal-to-ventral transition between areas 46 and p9/46v in anterior LPFC, while the pmfs-p appears to denote the dorsal-to-ventral transition between areas $8 \mathrm{Av}$ and $8 \mathrm{C}$ in posterior LPFC. Right, pmfs and imfs maximum probability maps relative to a resting-state fMRI parcellation with proposed homologous parcels between monkey and human LPFC from Sallet et al. (2013). Here, the pmfs-i and pmfs-a denote the 9/46d and 9/46v boundary, while the imfs is situated within area 46. This relationship is also consistent with a recent cytoarchitectonic atlas showing that the pmfs-a identifies a transition between 9/46v and 9/46d (Petrides, 2019).

anatomical theory (Sanides, 1962, 1964) as well as a recent cognitive neuroscience theory proposing a functional hierarchy in LPFC (Koechlin and Summerfield, 2007; Badre and D'Esposito, 2009; Badre and Nee, 2018). We end by discussing a growing need for computational tools that automatically define tertiary sulci throughout cortex.

The anatomical-functional coupling in LPFC identified here is quite surprising considering the widespread literature providing little support for fine-grained anatomical-functional coupling in this cortical expanse and in association cortices more broadly when conducting traditional group analyses (Paquola et al., 2019; Vázquez-Rodríguez et al., 2019). Indeed, cortical folding patterns relative to the location of anatomical, functional, or multimodal transitions are considered "imperfectly correlated" (Welker, 1990; Glasser et al., 2016) in association cortices and especially in LPFC (Van Essen et al., 2012; Caspers et al., 2013; Robinson et al., 2014; Coalson et al., 2018). Contrary to these previous findings that did not consider tertiary sulci, the present findings appear to support a classic, yet largely unconsidered theory proposed by Sanides $(1962,1964)$ that tertiary sulci are potentially meaningful anatomical and functional landmarks in association cortices-and in particular, in LPFC. Specifically, Sanides $(1962,1964)$ proposed that because tertiary sulci emerge late in gestation and exhibit a protracted postnatal development, they likely serve as functional and architectonic landmarks in human association cortices, which also exhibit a protracted postnatal development. Sanides (1964) further proposed that the late morphological development of tertiary sulci is likely related to protracted cognitive skills associated with LPFC. Interestingly, identifying pmfs components in his classic images shows myeloarchitectonic gradations among five areas in LPFC (Fig. 9a). Linking these data to recent modern parcellations of the human cerebral cortex (Sallet et al., 2013; Glasser et al., 2016) shows that pmfs components likely serve as boundaries 
among a series of cortical areas, which can be addressed in future research in individual participants (Fig. 9b).

In addition to supporting the classic anatomical theory of Sanides $(1962,1964)$, the present data demonstrated that the three pmfs components exhibit different resting-state connectivity profiles along a rostral-caudal axis, which builds on previous work also supporting a functional hierarchy along a rostral-caudal axis of LPFC. Further consistent with this hierarchy, evidence from neuroimaging, lesion, and electrocorticography studies indicate that this proposed rostral-caudal axis of LPFC is also related to levels of temporal and cognitive abstraction. That is, more anterior LPFC cortical regions are more highly engaged in tasks with higher abstract complexity (Koechlin et al., 2003; Koechlin and Summerfield, 2007; Voytek et al., 2015; Mansouri et al., 2017). While there are axonal tracing data in nonhuman primates suggesting an anatomical basis for such a hierarchical organization (Goulas et al., 2014, 2019), the present findings provide new evidence for anatomically and functionally dissociable sulcal components in LPFC that also support a hierarchical organization within individuals. Future work leveraging finer-scale multimodal and microanatomical data from individual human brains will be critical for uncovering anatomical and functional properties of LPFC across spatial and temporal scales that may further support the proposed functional rostral-caudal hierarchy of human LPFC.

Together, the culmination of present and previous findings suggest that tertiary sulci are landmarks in human ventral temporal cortex (Nasr et al., 2011; Caspers et al., 2013; Weiner et al., 2014; Lorenz et al., 2017), medial PFC (Amiez et al., 2019; Lopez-Persem et al., 2019), and, now, LPFC. This begs the following question: how many other tertiary sulci serve as cortical landmarks? We stress that it is unlikely that all tertiary sulci will serve as cortical landmarks, since neuroanatomists have known for over a century that not all sulci function as cortical landmarks (Smith, 1907; Bailey and Bonin, 1951; Ono et al., 1990; Welker, 1990; Van Essen et al., 2019). Nonetheless, this does not preclude the importance of future studies identifying which tertiary sulci are architectonic, functional, behavioral, or multimodal landmarks-not only in healthy young adults as examined here, but also in developmental (Voorhies et al., 2020) and clinical (Garrison et al., 2015; Brun et al., 2016; Rollins et al., 2020) cohorts. Additionally, tertiary sulci can also serve as evolutionary markers for primate cortical homology. For example, shallow "dimples" co-occur with the frontal eye field (FEF) in macaques, while deeper sulci co-occur with the proposed homolog of the FEF in humans (Amiez and Petrides, 2009; Schall et al., 2020). Humans may also have tertiary sulci in locations that nonhuman primates do not have dimples, as was recently shown in medial PFC (Amiez et al., 2019).

Carefully examining the relationship among tertiary sulci and multiple types of anatomical, functional, and behavioral data in individual participants will require new neuroimaging tools to automatically identify tertiary sulci throughout human cortex. For instance, most neuroimaging software packages are only capable of automatically defining $\sim 30-35$ primary and secondary sulci in a given hemisphere (Destrieux et al., 2010). Current estimates approximate $\sim 110$ sulci in each hemisphere when considering tertiary sulci (Petrides, 2019). Thus, studies in the immediate future will still require the manual identification of tertiary sulci, which is labor intensive and requires expertise (Miller et al., 2020a, for a historical discussion regarding the manual labeling of tertiary sulci in LPFC). For example, the present study required manual definitions of 936 sulci in 72 hemispheres.
While 72 is a large sample size compared with other labor-intensive anatomical studies in which 20 hemispheres is considered sufficient to encapsulate individual differences (Amunts and Zilles, 2015; Amunts et al., 2020), 2400 hemispheres are available just from the HCP alone. Defining tertiary sulci in only the LPFC of every HCP participant would require $\sim 26,400$ manual definitions, while defining all tertiary sulci in the entire HCP dataset would require over a quarter of a million $(\sim 256,800)$ manual definitions. Consequently, manual identification of tertiary sulci will continue to limit sample sizes in immediate future studies until new automated methods are generated (Klein et al., 2017; Hao et al., 2020; Lyu et al., 2020).

In the interim, we sought to leverage the anatomical labeling in this study to aid the field in the identification of sulcal landmarks in LPFC. The probability maps of sulcal locations in the present study are openly available and may be transformed to held-out individual brains (Fig. 9). Accordingly, manual identification of these landmarks within individuals is greatly aided, allowing future studies to apply these tools to identify LPFC tertiary in individual participants, including those from various groups such as patient or developmental cohorts. Because smaller tertiary sulci in association cortex are the latest sulcal indentations to develop (Sanides, 1962, 1964; Chi et al., 1977; Welker, 1990; Armstrong et al., 1995), their anatomical trajectories and properties likely relate to the development of cognitive abilities associated with the LPFC and other association areas as Sanides $(1962,1964)$ hypothesized, which recent ongoing work supports (Voorhies et al., 2020). Moving forward, we hope to leverage the manual labeling performed here to develop better automated algorithms for sulcal labeling within individuals. Future work using deep learning algorithms may help to identify tertiary structures in novel brains without manual labeling or intervention (Borne et al., 2020; Hao et al., 2020; Lyu et al., 2020). Such automated tools have translational applications as tertiary sulci are largely hominoid-specific structures (Amiez et al., 2019; Miller et al., 2020b) located in association cortices associated with pathology in many neurological disorders. Thus, morphological features of these understudied neuroanatomical structures may be useful clinical biomarkers for future diagnostic purposes. To begin to achieve this goal and to aid the field, we share our probabilistic maps of LPFC tertiary sulci with the publication of this article.

\section{References}

Amiez C, Petrides M (2007) Selective involvement of the mid-dorsolateral prefrontal cortex in the coding of the serial order of visual stimuli in working memory. Proc Natl Acad Sci U S A 104:13786-13791.

Amiez C, Petrides M (2009) Anatomical organization of the eye fields in the human and non-human primate frontal cortex. Prog Neurobiol 89:220230.

Amiez C, Sallet J, Hopkins WD, Meguerditchian A, Hadj-Bouziane F, Ben Hamed S, Wilson CRE, Procyk E, Petrides M (2019) Sulcal organization in the medial frontal cortex provides insights into primate brain evolution. Nat Commun 10:3437.

Amunts K, Zilles K (2015) Architectonic mapping of the human brain beyond Brodmann. Neuron 88:1086-1107.

Amunts K, Mohlberg H, Bludau S, Zilles K (2020) Julich-Brain: a 3D probabilistic atlas of the human brain's cytoarchitecture. Science 369:988-992.

Annese J, Pitiot A, Dinov ID, Toga AW (2004) A myelo-architectonic method for the structural classification of cortical areas. Neuroimage 21:15-26.

Armstrong E, Schleicher A, Omran H, Curtis M, Zilles K (1995) The ontogeny of human gyrification. Cereb Cortex 5:56-63.

Badre D, D'Esposito M (2009) Is the rostro-caudal axis of the frontal lobe hierarchical? Nat Rev Neurosci 10:659-669. 
Badre D, Nee DE (2018) Frontal cortex and the hierarchical control of behavior. Trends Cogn Sci 22:170-188.

Bailey P, Bonin GV (1951) The isocortex of man. Urbana, IL: University of Illinois.

Barrett RLC, Dawson M, Dyrby TB, Krug K, Ptito M, D’Arceuil H, Croxson PL, Johnson PJ, Howells H, Forkel SJ, Dell'Acqua F, Catani M (2020) Differences in frontal network anatomy across primate species. J Neurosci 40:2094-2107.

Benson NC, Jamison KW, Arcaro MJ, Vu AT, Glasser MF, Coalson TS, Van Essen DC, Yacoub E, Ugurbil K, Winawer J, Kay K (2018) The Human Connectome Project 7 Tesla retinotopy dataset: description and population receptive field analysis. J Vis 18:23.

Borne L, Rivière D, Mancip M, Mangin JF (2020) Automatic labeling of cortical sulci using patch- or CNN-based segmentation techniques combined with bottom-up geometric constraints. Med Image Anal 62:101651.

Braitenberg V (1962) A note on myeloarchitectonics. J Comp Neurol 118:141-156.

Brun L, Auzias G, Viellard M, Villeneuve N, Girard N, Poinso F, Da Fonseca D, Deruelle C (2016) Localized misfolding within broca's area as a distinctive feature of autistic disorder. Biol Psychiatry Cogn Neurosci Neuroimaging 1:160-168.

Caspers J, Zilles K, Eickhoff SB, Schleicher A, Mohlberg H, Amunts K (2013) Cytoarchitectonical analysis and probabilistic mapping of two extrastriate areas of the human posterior fusiform gyrus. Brain Struct Funct 218:511526.

Chi JG, Dooling EC, Gilles FH (1977) Gyral development of the human brain. Ann Neurol 1:86-93.

Coalson TS, Van Essen DC, Glasser MF (2018) The impact of traditional neuroimaging methods on the spatial localization of cortical areas. Proc Natl Acad Sci U S A 115:E6356-E6365.

Connolly C (1950) External morphology of the primate brain. Am J Phys Anthropol 8:516-517.

Dale AM, Fischl B, Sereno MI (1999) Cortical surface-based analysis. I. Segmentation and surface reconstruction. Neuroimage 9:179-194.

Demirtaş M, Burt JB, Helmer M, Ji JL, Adkinson BD, Glasser MF, Van Essen DC, Sotiropoulos SN, Anticevic A, Murray JD (2019) Hierarchical heterogeneity across human cortex shapes large-scale neural dynamics. Neuron 101:1181-1194.e13.

Destrieux C, Fischl B, Dale A, Halgren E (2010) Automatic parcellation of human cortical gyri and sulci using standard anatomical nomenclature. Neuroimage 53:1-15.

Dick F, Tierney AT, Lutti A, Josephs O, Sereno MI, Weiskopf N (2012) In vivo functional and myeloarchitectonic mapping of human primary auditory areas. J Neurosci 32:16095-16105.

Ding S-L, Royall JJ, Sunkin SM, Ng L, Facer BAC, Lesnar P, GuillozetBongaarts A, McMurray B, Szafer A, Dolbeare TA, Stevens A, Tirrell L, Benner T, Caldejon S, Dalley RA, Dee N, Lau C, Nyhus J, Reding M, Riley ZL, et al (2016) Comprehensive cellular-resolution atlas of the adult human brain. J Comp Neurol 524:3127-3481.

Donahue CJ, Glasser MF, Preuss TM, Rilling JK, Van Essen DC (2018) Quantitative assessment of prefrontal cortex in humans relative to nonhuman primates. Proc Natl Acad Sci U S A 115:E5183-E5192.

Eberstaller O (1890) Das stirnhirn. Vienna: Urban \& Schwarzenberg.

Fischl B, Sereno MI, Dale AM (1999a) Cortical surface-based analysis. II: inflation, flattening, and a surface-based coordinate system. Neuroimage 9:195-207.

Fischl B, Sereno MI, Tootell RBH, Dale AM (1999b) High-resolution intersubject averaging and a coordinate system for the cortical surface. Hum Brain Mapp 8:272-284.

Fisher AV (2019) Selective sustained attention: a developmental foundation for cognition. Curr Opin Psychol 29:248-253.

Flechsig P (1920) Anatomie des menschlichen gehirns und rückenmarks auf myelogenetischer grundlage. Leipzig, Germany: G. Thieme.

Fritz CO, Morris PE, Richler JJ (2012) Effect size estimates: current use, calculations, and interpretation. J Exp Psychol Gen 141:2-18.

Gao JS, Huth AG, Lescroart MD, Gallant JL (2015) Pycortex: an interactive surface visualizer for fMRI. Front Neuroinform 9:23.

Garrison JR, Fernyhough C, McCarthy-Jones S, Haggard M, Australian Schizophrenia Research B, Simons JS (2015) Paracingulate sulcus morphology is associated with hallucinations in the human brain. Nat Commun 6:8956.
Germann J, Petrides M (2020) Area 8A within the posterior middle frontal gyrus underlies cognitive selection between competing visual targets. eNeuro 7:ENEURO.0102-20.2020.

Glasser MF, Van Essen DC (2011) Mapping human cortical areas in vivo based on myelin content as revealed by T1- and T2-weighted MRI. J Neurosci 31:11597-11616.

Glasser MF, Sotiropoulos SN, Wilson JA, Coalson TS, Fischl B, Andersson JL, Xu J, Jbabdi S, Webster M, Polimeni JR, Van Essen DC, Jenkinson M (2013) The minimal preprocessing pipelines for the Human Connectome Project. Neuroimage 80:105-124.

Glasser MF, Coalson TS, Robinson EC, Hacker CD, Harwell J, Yacoub E, Ugurbil K, Andersson J, Beckmann CF, Jenkinson M, Smith SM, Van Essen DC (2016) A multi-modal parcellation of human cerebral cortex. Nature 536:171-178.

Gordon EM, Laumann TO, Gilmore AW, Newbold DJ, Greene DJ, Berg JJ, Ortega M, Hoyt-Drazen C, Gratton C, Sun H, Hampton JM, Coalson RS, Nguyen AL, McDermott KB, Shimony JS, Snyder AZ, Schlaggar BL, Petersen SE, Nelson SM, Dosenbach NUF (2017) Precision functional mapping of individual human brains. Neuron 95:791-807.e7.

Goulas A, Uylings HB, Stiers P (2014) Mapping the hierarchical layout of the structural network of the macaque prefrontal cortex. Cereb Cortex 24:1178-1194

Goulas A, Majka P, Rosa MGP, Hilgetag CC (2019) A blueprint of mammalian cortical connectomes. PLoS Biol 17:e2005346.

Hagler DJ Jr, Sereno MI (2006) Spatial maps in frontal and prefrontal cortex. Neuroimage 29:567-577.

Hao L, Bao S, Tang Y, Gao R, Parvathaneni P, Miller JA, Voorhies W, Yao J, Bunge SA, Weiner KS, Landman BA, Lyu I (2020) Automatic labeling of cortical sulci using convolutional neural networks in a developmental cohort. In: IEEE ISBI 2020, International Symposium on Biomedical Imaging: 2020 workshop proceedings. Piscataway, NJ: IEEE.

Hinds OP, Rajendran N, Polimeni JR, Augustinack JC, Wiggins G, Wald LL, Diana Rosas H, Potthast A, Schwartz EL, Fischl B (2008) Accurate prediction of V1 location from cortical folds in a surface coordinate system. Neuroimage 39:1585-1599.

Hopf A (1956) Ûber die Verteilung myeloarchitektonischer Merkmale in der Stirnhirnrinde beim Menschen. J Hirnforsch 2:311-333.

Kastner S, DeSimone K, Konen CS, Szczepanski SM, Weiner KS, Schneider KA (2007) Topographic maps in human frontal cortex revealed in memory-guided saccade and spatial working-memory tasks. J Neurophysiol 97:3494-3507.

Klein A, Ghosh SS, Bao FS, Giard J, Häme Y, Stavsky E, Lee N, Rossa B, Reuter M, Chaibub Neto E, Keshavan A (2017) Mindboggling morphometry of human brains. PLoS Comput Biol 13:e1005350.

Koechlin E, Summerfield C (2007) An information theoretical approach to prefrontal executive function. Trends Cogn Sci 11:229-235.

Koechlin E, Ody C, Kouneiher F (2003) The architecture of cognitive control in the human prefrontal cortex. Science 302:1181-1185.

Kong R, Li J, Orban C, Sabuncu MR, Liu H, Schaefer A, Sun N, Zuo XN, Holmes AJ, Eickhoff SB, Yeo BTT (2018) Spatial topography of individual-specific cortical networks predicts human cognition, personality, and emotion. Cereb Cortex 28:3095-3114.

Lopez-Persem A, Verhagen L, Amiez C, Petrides M, Sallet J (2019) The human ventromedial prefrontal cortex: sulcal morphology and its influence on functional organization. J Neurosci 39:3627-3639.

Lorenz S, Weiner KS, Caspers J, Mohlberg H, Schleicher A, Bludau S, Eickhoff SB, Grill-Spector K, Zilles K, Amunts K (2017) Two new cytoarchitectonic areas on the human mid-fusiform gyrus. Cereb Cortex 27:373-385.

Lyu I, Bao S, Hao L, Yao J, Miller JA, Voorhies W, Taylor WD, Bunge SA, Weiner KS, Landman BA (2020) Labeling lateral prefrontal sulci using spherical data augmentation and context-aware training. Neuroimage, in press.

Mackey WE, Winawer J, Curtis CE (2017) Visual field map clusters in human frontoparietal cortex. Elife 6:e22974.

Madan CR (2019) Robust estimation of sulcal morphology. Brain Inform 6:5.

Mansouri FA, Koechlin E, Rosa MGP, Buckley MJ (2017) Managing competing goals - a key role for the frontopolar cortex. Nat Rev Neurosci 18:645-657.

Miller EK, Cohen JD (2001) An integrative theory of prefrontal cortex function. Annu Rev Neurosci 24:167-202. 
Miller JA, D'Esposito M, Weiner KS (2020a) Using tertiary sulci to map the "cognitive globe" of prefrontal cortex. psyArxiv. doi: 10.31234/osf.io/ y8f4b.

Miller JA, Voorhies WI, Li X, Raghuram I, Palomero-Gallagher N, Zilles K, Sherwood CC, Hopkins WD, Weiner KS (2020b) Sulcal morphology of ventral temporal cortex is shared between humans and other hominoids. Sci Rep 10:17132.

Nasr S, Liu N, Devaney KJ, Yue X, Rajimehr R, Ungerleider LG, Tootell RB (2011) Scene-selective cortical regions in human and nonhuman primates. J Neurosci 31:13771-13785.

Nee DE, D'Esposito M (2016) The hierarchical organization of the lateral prefrontal cortex. eLife 5:e12112.

Ono M, Kubik S, Abernathey C (1990) Atlas of the cerebral sulci. New York: Thieme Medical Publishers.

Paquola C, Vos De Wael R, Wagstyl K, Bethlehem RAI, Hong SJ, Seidlitz J, Bullmore ET, Evans AC, Misic B, Margulies DS, Smallwood J, Bernhardt BC (2019) Microstructural and functional gradients are increasingly dissociated in transmodal cortices. PLoS Biol 17:e3000284.

Petrides M (2019) Atlas of the morphology of the human cerebral cortex on the average MNI brain, Ed 1. London, UK: Elsevier.

Petrides M, Pandya DN (2012) The-frontal-cortex. In: The human nervous system (Mai J, Paxinos G, eds), pp 988-1011. Amsterdam: Elsevier.

Rajkowska G, Goldman-Rakic PS (1995) Cytoarchitectonic definition of prefrontal areas in the normal human cortex: II. Variability in locations of areas 9 and 46 and relationship to the Talairach coordinate system. Cereb Cortex 5:323-337.

Retzius G (1896) Das menschenhirn. Stockholm, Sweden: Norstedt and Soener.

Robinson EC, Jbabdi S, Glasser MF, Andersson J, Burgess GC, Harms MP, Smith SM, Van Essen DC, Jenkinson M (2014) MSM: a new flexible framework for multimodal surface matching. Neuroimage 100:414-426.

Rollins CPE, Garrison JR, Arribas M, Seyedsalehi A, Li Z, Chan RCK, Yang J, Wang D, Liò P, Yan C, Yi Z-H, Cachia A, Upthegrove R, Deakin B, Simons JS, Murray GK, Suckling J (2020) Evidence in cortical folding patterns for prenatal predispositions to hallucinations in schizophrenia. Transl Psychiatry 10:387.

Rowley CD, Bazin PL, Tardif CL, Sehmbi M, Hashim E, Zaharieva N, Minuzzi L, Frey BN, Bock NA (2015) Assessing intracortical myelin in the living human brain using myelinated cortical thickness. Front Neurosci 9:396.

Sallet J, Mars RB, Noonan MP, Neubert FX, Jbabdi S, O'Reilly JX, Filippini N, Thomas AG, Rushworth MF (2013) The organization of dorsal frontal cortex in humans and macaques. J Neurosci 33:12255-12274.

Sanides F (1962) Die architektonik des menschlichen stirnhirns zugleich eine darstellung der prin. In: Monographien aus dem gesamtgebiete der neurologie und psychiatrie (Muller M, Spatz H, Vogel P, eds), pp 176-190. Berlin: Springer.

Sanides F (1964) Structure And Function Of The Human Frontal Lobe. Neuropsychologia 2:209-219.

Sanides F (1972) Representation in the cerebral cortex and its areal lamination patterns. In: The structure and function of nervous tissue (Bourne GH, ed), pp 329-453. New York: Academic.

Schall JD, Zinke W, Cosman JD, Schall MS, Pare M, Pouget P (2020) On the evolution of the frontal eye field: comparisons of monkeys, apes, and humans. In: Evolutionary neuroscience, Ed 2 (Kaas JH, ed), pp 861-883. Amsterdam: Elsevier.

Seitzman BA, Gratton C, Laumann TO, Gordon EM, Adeyemo B, Dworetsky A, Kraus BT, Gilmore AW, Berg JJ, Ortega M, Nguyen A, Greene DJ, McDermott KB, Nelson SM, Lessov-Schlaggar CN, Schlaggar BL, Dosenbach NUF, Petersen SE (2019) Trait-like variants in human functional brain networks. Proc Natl Acad Sci U S A 116:22851-22861.
Semendeferi K, Lu A, Schenker N, Damasio H (2002) Humans and great apes share a large frontal cortex. Nat Neurosci 5:272-276.

Shams Z, Norris DG, Marques JP (2019) A comparison of in vivo MRI based cortical myelin mapping using T1w/T2w and R1 mapping at 3T. PLoS One 14:e0218089.

Smith GE (1907) A new topographical survey of the human cerebral cortex, being an account of the distribution of the anatomically distinct cortical areas and their relationship to the cerebral sulci. J Anat Physiol 41:237254.

Szczepanski SM, Knight RT (2014) Insights into human behavior from lesions to the prefrontal cortex. Neuron 83:1002-1018.

Van Essen DC, Dierker DL (2007) Surface-based and probabilistic atlases of primate cerebral cortex. Neuron 56:209-225.

Van Essen DC, Glasser MF, Dierker DL, Harwell J (2012) Cortical parcellations of the macaque monkey analyzed on surface-based atlases. Cereb Cortex 22:2227-2240.

Van Essen DC, Donahue CJ, Coalson TS, Kennedy H, Hayashi T, Glasser MF (2019) Cerebral cortical folding, parcellation, and connectivity in humans, nonhuman primates, and mice. Proc Natl Acad Sci U S A 116:26173-26180

Vázquez-Rodríguez B, Suárez LE, Markello RD, Shafiei G, Paquola C, Hagmann P, van den Heuvel MP, Bernhardt BC, Spreng RN, Misic B (2019) Gradients of structure-function tethering across neocortex. Proc Natl Acad Sci U S A 116:21219-21227.

Vogt C, Vogt O (1919) Allgemeinere ergebnisse unserer hirnforschung. J Psychol Neurol 25:279-462.

Voorhies W, Miller JA, Yao J, Bunge SA, Weiner KS (2020) Cognitive insights from evolutionarily new brain structures in prefrontal cortex. bioRxiv. doi: 10.1101/2020.11.07.372805.

Voytek B, Kayser AS, Badre D, Fegen D, Chang EF, Crone NE, Parvizi J, Knight RT, D'Esposito M (2015) Oscillatory dynamics coordinating human frontal networks in support of goal maintenance. Nat Neurosci 18:1318-1324.

Waehnert MD, Dinse J, Weiss M, Streicher MN, Waehnert P, Geyer S, Turner R, Bazin PL (2014) Anatomically motivated modeling of cortical laminae. Neuroimage 93:210-220.

Wang L, Mruczek RE, Arcaro MJ, Kastner S (2015) Probabilistic maps of visual topography in human cortex. Cereb Cortex 25:3911-3931.

Weiner KS, Natu VS, Grill-Spector K (2018) On object selectivity and the anatomy of the human fusiform gyrus. Neuroimage 173:604-609.

Weiner KS, Golarai G, Caspers J, Chuapoco MR, Mohlberg H, Zilles K, Amunts K, Grill-Spector K (2014) The mid-fusiform sulcus: a landmark identifying both cytoarchitectonic and functional divisions of human ventral temporal cortex. Neuroimage 84:453-465.

Weiner KS, Barnett MA, Lorenz S, Caspers J, Stigliani A, Amunts K, Zilles K, Fischl B, Grill-Spector K (2017) The cytoarchitecture of domain-specific regions in human high-level visual cortex. Cereb Cortex 27:146-161.

Welker W (1990) Why does cerebral cortex fissure and fold? In: A review of determinants of gyri and sulci (Peters A, Jones EG, eds), pp 3-136. New York: Plenum.

Yeo BT, Krienen FM, Sepulcre J, Sabuncu MR, Lashkari D, Hollinshead M, Roffman JL, Smoller JW, Zöllei L, Polimeni JR, Fischl B, Liu H, Buckner RL (2011) The organization of the human cerebral cortex estimated by intrinsic functional connectivity. J Neurophysiol 106:1125-1165.

Yeo BT, Krienen FM, Eickhoff SB, Yaakub SN, Fox PT, Buckner RL, Asplund CL, Chee MW (2015) Functional specialization and flexibility in human association cortex. Cereb Cortex 25:3654-3672.

Zilles K, Palomero-Gallagher N, Amunts K (2013) Development of cortical folding during evolution and ontogeny. Trends Neurosci 36:275-284. 